\title{
Towards a census of the Galactic anticentre star clusters: colour-magnitude diagram and structural analyses of a sample of 50 objects
}

\author{
D. Camargo, C. Bonatto, and E. Bica
}

\begin{abstract}
Universidade Federal do Rio Grande do Sul, Departamento de Astronomia, CP 15051, RS, Porto Alegre 91501-970, Brazil e-mail: denilso.camargo@ufrgs.br; [charles; bica]@if.ufrgs.br
\end{abstract}

Received 8 April 2010 / Accepted 13 June 2010

\section{ABSTRACT}

\begin{abstract}
Aims. In this work we investigate the nature of 50 overdensities from the catalogue of Froebrich, Scholz, and Raftery (FSR) projected towards the Galactic anticentre, in the sector $160^{\circ} \leq \ell \leq 200^{\circ}$. The sample contains candidates with $|b| \leq 20^{\circ}$ classified by FSR as probable open cluster (OC) and labelled with quality flags 2 and 3. Our main purpose is to determine the nature of these OC candidates and the fraction of these objects that are unknown OCs, as well as to derive astrophysical parameters (age, reddening, distance, core and cluster radii) for the clusters and to investigate the relationship among parameters.

Methods. The analysis is based on 2MASS $J,(J-H)$, and $\left(J-K_{\mathrm{s}}\right)$ colour-magnitude diagrams (CMDs), and stellar radial density profiles (RDPs) built with decontamination tools. The tools are a field star decontamination algorithm, used to uncover the cluster's intrinsic CMD morphology, and colour-magnitude filters to isolate stars with a high probability of being cluster members.

Results. Out of the 50 objects, $16(32 \%)$ are star clusters. We show that $9(18 \%)$ overdensities are new OCs (FSR 735, FSR 807, FSR 812, FSR 826, FSR 852, FSR 904, FSR 941, FSR 953, and FSR 955) and 7 (14\%) are previously studied or catalogued OCs (KKC1, FSR 795, Cz 22, FSR 828, FSR 856, Cz 24, and NGC 2234). These are OCs with ages in the range $5 \mathrm{Myr}$ to $1 \mathrm{Gyr}$, at distances from the Sun $1.28 \lesssim d_{\odot}(\mathrm{kpc}) \lesssim 5.78$ and Galactocentric distances $8.5 \lessgtr R_{\mathrm{GC}}(\mathrm{kpc}) \lessgtr 12.9$. We also derive parameters for the previously analysed OCs $\mathrm{Cz} 22$ and NGC 2234. Five (10\%) candidates are classified as uncertain cases, and the remaining objects are probable field fluctuations.
\end{abstract}

Key words. galaxies: clusters: general - Galaxy: stellar content - Galaxy: structure

\section{Introduction}

It is currently accepted that star formation occurs mainly in clustered environments, such as clusters and associations, rather than in isolation. However, only a very small fraction of old stars are found in bound clusters (Lamers et al. 2005; Lamers \& Gieles 2006). On the other hand, de Wit et al. (2005) estimate that nearly $95 \%$ of the Galactic $\mathrm{O}$ star population is located in clusters or $\mathrm{OB}$ associations, or can be kinematically linked with them. In this sense, these structures can be thought of as the fundamental units of star formation in the Galaxy (Lada 2007).

The spatial and age distribution of clusters has also played a vital role in our understanding of the Galactic structure. In this context, young open clusters (OCs) are important tracers of recent star formation in galaxies and of the spiral structure in galactic disks (Lada \& Lada 2003). On the other hand, old OCs are excellent probes of early disk evolution, and they provide tracers of the structure, kinematics, and chemistry of the Galactic disk (Friel 1995).

Primordial conditions during cluster formation and the location of the parental molecular cloud in the Galaxy play an important role in the fate of a cluster (Schilbach et al. 2006). However, the stellar content of a cluster evolves with time, and internal and external interactions affect the properties of individual cluster members (e.g. orbit and spatial location), as well as of the whole cluster as a system (e.g. mass and structure). A thorough review of young clusters, focussing particularly on the evolution of the massive ones, can be found in Portegies Zwart et al. (2010).
The age distribution of star clusters in the Galactic disk can only be explained if they disrupt on a timescale of a few times $10^{8} \mathrm{yr}$ (Lamers \& Gieles 2006, and references therein). Lada \& Lada (2003) estimate that, in the solar neighbourhood, less than $\sim 4 \%$ of the clusters formed in giant molecular clouds (GMCs) are able to reach ages beyond $100 \mathrm{Myr}$, and less than $10 \%$ survive longer than $\sim 10 \mathrm{Myr}$ (infant mortality). Oort (1957) noticed that the distribution of OCs in the solar neighbourhood, as a function of age, shows a lack of old OCs and derived statistically that Galactic clusters disrupt on a timescale of $5 \times 10^{8} \mathrm{yr}$. This lack of old clusters can be partially explained by the rapid fading of clusters with age due to stellar evolution, which makes it harder to observe them at older ages. However, fading cannot explain the difference between the observed and the expected number of old OCs, implying that a significant fraction must have been destroyed. Wielen (1971) derived a mean dissolution time of $200 \mathrm{Myr}$ from the age distribution of clusters with mass range $10^{2}-10^{3} M_{\odot}$ about $1 \mathrm{kpc}$ from the Sun (see also Lamers \& Gieles 2006; Gieles et al. 2007).

Star clusters gradually lose mass and, because of the combined effect of several disruption mechanisms ${ }^{1}$, they generally end completely destroyed or leave remnants (Pavani \& Bica 2007, and references therein). As a consequence, only the more massive OCs (which are essentially gravitationally bound) and

\footnotetext{
${ }^{1}$ E.g., mass loss by stellar evolution, mass segregation, and tidal interactions with the disk, bulge, spiral arms and GMCs. The latter events increase the mean internal energy that may lead to the gradual cluster expansion and disruption.
} 
those located at large Galactic radii (where the probability of encounters with GMCs is lower) can live a few Gyr (Bergond et al. 2001).

Most of the young star clusters dissolve very early because the primordial gas is removed on a timescale shorter than a crossing time, by winds of massive OB stars and supernova explosions (Tutukov 1978). This mechanism strongly depends on the effective star formation efficiency, but appears to be independent of the initial cluster mass (Gieles et al. 2005).

Open clusters are born within GMCs and remain embedded in the clouds for about 2-5 Myr. During formation and the earliest stages of evolution, they are often completely invisible at optical wavelengths, since they are only detected in the infrared because of heavy obscuration by gas and dust (Lada \& Lada 2003). The recent development of infrared array detectors has provided an important impulse to our knowledge of these objects.

In the present work we investigate the nature of 50 stellar overdensities from the catalogue of Froebrich et al. (2007) towards the Galactic anticentre, in the sector $\left(160^{\circ} \leq \ell \leq 200^{\circ}\right)$, classified by them as probable OC and labelled with quality flags 2 and 3 (Table 1).

This paper is organised as follows. In Sect. 2 we present the OC candidates. In Sect. 3 we present the 2MASS photometry and discuss the methods and tools employed in the CMD analyses, especially the field star decontamination algorithm. Section 4 is dedicated to the discussion of the methods and tools used for the analysis of cluster structure. In Sect. 5 we present the results of our analyses of the OC candidates, and derive astrophysical parameters (age, reddening, distance, core and cluster radii) of the confirmed OCs and two previously studied objects. In Sect. 6 we estimate the cluster mass stored in stars. In Sect. 7 we discuss the results and investigate the relationship between derived parameters. Finally, in Sect. 8 we present concluding remarks.

\section{The $O C$ candidates}

Froebrich et al. (2007) have published a catalogue of 1021 star cluster candidates with $|b| \leq 20^{\circ}$ and all Galactic longitudes. They identify overdensities in the 2MASS database that are classified according to a quality flag, with 0 and 1 for the most probable star clusters and 2-5 for possible star clusters. Bica et al. (2008a) explored FSR overdensities, with quality flags 0 and 1 , in bulge/disk directions at $|\ell| \leq 60^{\circ}$. The sample consisted of 20 star cluster candidates and resulted in 4 new, 2 previously known OCs, 5 uncertain cases, and 9 probable field fluctuations. Bonatto \& Bica (2008b) analysed 28 FSR cluster candidates projected nearly towards the anti-centre $\left(160^{\circ} \leq \ell \leq 200^{\circ}\right)$ and confirm 6 new and 9 previously known OCs, 6 uncertain cases, and 7 probable fluctuations of the stellar field.

The present FSR overdensity sample is listed in Table 1. Some of these objects have previous identifications (Table 2). The Koposov et al. (2008) analysis of CMDs for 5 clusters is showed in Table 2. They concluded that FSR 795 (Koposov 10), Cz 22, FSR 828 (Koposov 43), FSR 856 (Koposov 53) and Cz 24 are clusters. The derived parameters for them and the present ones (Tables 3 and 4) agree well. Kumar et al. (2006) studied KKC1 (FSR 788) and found that it is a cluster (Sect. 5). We derive parameters for the previously catalogued OCs $\mathrm{Cz} 22$ and NGC 2234. Yadav \& Sagar (2004) derived for $\mathrm{Cz} 22$ a radius of $1.8^{\prime}$, metallicity of $0.08, E(B-V)=0.45 \pm 0.05$, age of the $200 \pm 50 \mathrm{Myr}$ and $d_{\odot}=3.0 \pm 0.2 \mathrm{kpc}$. NGC2234 does not have parameters derived so far.

Page 2 of 13
Table 1. General data on the FSR star cluster candidates.

\begin{tabular}{|c|c|c|c|c|c|c|c|}
\hline Target & $\begin{array}{r}\alpha(2000) \\
(\mathrm{h} \mathrm{m} \mathrm{s}) \\
(2)\end{array}$ & $\begin{array}{r}\delta(2000) \\
\left({ }^{\prime \prime} \prime \prime\right) \\
(3)\end{array}$ & $\begin{array}{r}\ell \\
\left({ }^{\circ}\right) \\
(4)\end{array}$ & $\begin{array}{r}b \\
\left({ }^{\circ}\right) \\
(5)\end{array}$ & $\begin{array}{l}R_{\mathrm{C}} \\
(') \\
(6)\end{array}$ & $\begin{array}{r}R_{t} \\
(') \\
(7)\end{array}$ & (8) \\
\hline FSR 714 & $04: 42: 46$ & $41: 55: 19$ & 162.024 & -2.745 & 1.32 & 6.66 & 2 \\
\hline FSR 717 & 04:46:06 & 42:08:03 & 162.267 & -2.138 & 1.08 & 53.7 & 2 \\
\hline FSR 719 & $04: 44: 19$ & $41: 48: 51$ & 162.295 & -2.597 & 1.32 & 18.12 & 0 \\
\hline FSR 721 & $04: 46: 21$ & $41: 57: 07$ & 162.436 & -2.221 & 2.82 & 5.64 & 3 \\
\hline FSR 723 & $04: 44: 57$ & $41: 30: 45$ & 162.602 & -2.703 & 0.42 & 4.56 & 3 \\
\hline FSR 732 & $05: 02: 36$ & $42: 32: 55$ & 163.867 & +0.49 & 1.26 & 4.98 & 2 \\
\hline FSR 733 & 04:45:08 & $39: 46: 32$ & 163.945 & -3.808 & 1.98 & 5.88 & 3 \\
\hline FSR 735 & $04: 53: 57$ & $40: 50: 03$ & .208 & -1.84 & 2.34 & 7.02 & 2 \\
\hline FSR 753 & $05: 02: 14$ & $36: 47: 24$ & 168.385 & -3.084 & 0.96 & 10.38 & 2 \\
\hline FSR 775 & $05: 25: 35$ & $34: 57: 27$ & 172.641 & -0.32 & 3.18 & 6.42 & 3 \\
\hline FSR 778 & $05: 14: 20$ & $32: 47: 43$ & 173.079 & -3.474 & 0.3 & 1.56 & 3 \\
\hline FSR $788^{\dagger}$ & $05: 41: 30$ & $35: 48: 39$ & 173.688 & +2.865 & 0.78 & 37.5 & 3 \\
\hline FSR 795 & $05: 47: 29$ & $35: 25: 56$ & 174.647 & +3.707 & 1.08 & 54.6 & 2 \\
\hline FSR 807 & $05: 36: 39$ & $31: 49: 20$ & 176.529 & -0.111 & 2.04 & 16.56 & 2 \\
\hline FSR 812 & 05:38:11 & $31: 44: 03$ & 176.777 & +0.115 & 1.26 & 3.84 & 2 \\
\hline FSR 815 & $05: 37: 42$ & $31: 19: 27$ & 177.069 & -0.19 & 1.44 & 4.38 & 2 \\
\hline FSR 821 & $05: 41: 53$ & $29: 54: 17$ & 178.749 & -0.184 & 4.98 & 9.96 & 3 \\
\hline FSR $825^{\dagger}$ & $05: 48: 57$ & $30: 10: 24$ & 179.317 & +1.261 & 0.66 & 33.36 & 2 \\
\hline FSR 826 & $05: 42: 52$ & $28: 56: 29$ & 179.681 & -0.509 & 0.96 & 3.90 & 2 \\
\hline FSR 828 & $05: 52: 18$ & $29: 54: 16$ & 179.919 & +1.746 & 1.38 & 43.56 & 2 \\
\hline FSR 852 & $05: 53: 35$ & $25: 10: 52$ & 184.133 & -0.408 & 1.56 & 6.30 & 3 \\
\hline FSR 856 & 06:08:55 & $26: 15: 59$ & 184.899 & +3.13 & 0.54 & 11.04 & 2 \\
\hline FSR 858 & 06:09:09 & $25: 40: 38$ & 185.441 & +2.891 & 0.9 & 41.28 & 2 \\
\hline FSR $881^{\dagger}$ & $05: 55: 25$ & $20: 52: 59$ & 188.056 & -2.216 & 0.6 & 31.02 & 3 \\
\hline FSR 883 & 06:04:20 & $22: 00: 52$ & 188.106 & +0.147 & 1.62 & 81.42 & 2 \\
\hline FSR 891 & $06: 17: 29$ & $22: 25: 38$ & 189.209 & 3.011 & 0.72 & 5.76 & 2 \\
\hline FSR 900 & $06: 06: 30$ & $19: 13: 59$ & 190.78 & -0.771 & 0.3 & 1.50 & 3 \\
\hline FSR 902 & $06: 18: 19$ & $20: 31: 42$ & 190.978 & +2.288 & 0.6 & 31.5 & 3 \\
\hline FSR 903 & $06: 07: 34$ & $19: 06: 46$ & 191.007 & -0.611 & 0.66 & 21.24 & 2 \\
\hline FSR 904 & 06:07:00 & 19:00:43 & 191.03 & -0.777 & 1.38 & 57.84 & 2 \\
\hline FSR 906 & $06: 07: 44$ & $18: 47: 26$ & 191.308 & -0.733 & 2.16 & 6.42 & 3 \\
\hline FSR 909 & $06: 15: 46$ & 19:00:49 & 192.026 & +1.039 & 0.24 & 1.56 & 3 \\
\hline FSR 921 & $06: 05: 17$ & $16: 40: 41$ & 192.87 & -2.27 & 1.14 & 55.5 & 2 \\
\hline FSR 924 & $06: 10: 26$ & $16: 50: 13$ & 193.329 & -1.116 & 1.38 & 5.58 & 3 \\
\hline FSR 928 & 06:04:20 & $15: 24: 50$ & 193.861 & -3.09 & 0.84 & 40.5 & 3 \\
\hline FSR 931 & $06: 13: 07$ & $15: 56: 37$ & 194.422 & -0.981 & 0.3 & 7.14 & 3 \\
\hline FSR 933 & $06: 05: 37$ & $14: 32: 07$ & 194.781 & -3.247 & 4.32 & 8.70 & 3 \\
\hline FSR 936 & $06: 28: 31$ & $16: 55: 36$ & 195.293 & +2.737 & 0.66 & 33.2 & 3 \\
\hline FSR 937 & $06: 13: 41$ & $14: 59: 47$ & 195.319 & -1.315 & 0.48 & 2.94 & 2 \\
\hline FSR $938^{\dagger}$ & 06:29:20 & $16: 45: 27$ & 195.535 & +2.835 & 1.26 & 3.8 & 2 \\
\hline FSR 939 & $06: 10: 57$ & $14: 22: 57$ & 195.54 & -2.191 & 0.9 & 46.0 & 3 \\
\hline FSR 940 & $06: 14: 16$ & $14: 48: 18$ & 195.554 & -1.284 & 0.7 & 37.1 & 2 \\
\hline FSR 941 & $06: 21: 51$ & $15: 45: 59$ & 195.574 & +0.782 & 3.0 & 9.0 & 2 \\
\hline FSR 951 & $06: 22: 19$ & $14: 42: 44$ & 196.558 & +0.387 & 0.4 & 22.4 & 2 \\
\hline FSR 952 & $06: 10: 15$ & $13: 00: 47$ & 196.661 & -2.997 & 0.8 & 10.6 & 3 \\
\hline FSR 953 & $06: 19: 02$ & $14: 08: 53$ & 196.681 & -0.58 & 0.4 & 8.8 & 2 \\
\hline FSR 955 & $06: 23: 52$ & $14: 30: 26$ & 196.915 & +0.622 & 0.2 & 12.5 & 3 \\
\hline FSR 960 & 06:09:53 & $12: 20: 02$ & 197.215 & -3.401 & 2.3 & 4.6 & 3 \\
\hline FSR 962 & $06: 23: 50$ & $14: 05: 10$ & 197.284 & +0.419 & 0.8 & 23.8 & 3 \\
\hline FSR 975 & $06: 40: 00$ & $13: 17: 58$ & 199.795 & +3.54 & 2.3 & 4.5 & 2 \\
\hline
\end{tabular}

Notes. Columns 2-3: Central coordinates provided by Froebrich et al. (2007). Columns 4-5: Corresponding Galactic coordinates. Columns 6-7: Core and tidal radii derived by Froebrich et al. (2007) from King fits. Column 8: FSR quality flag. $\dagger$ : has a previous designation (Table 2). 

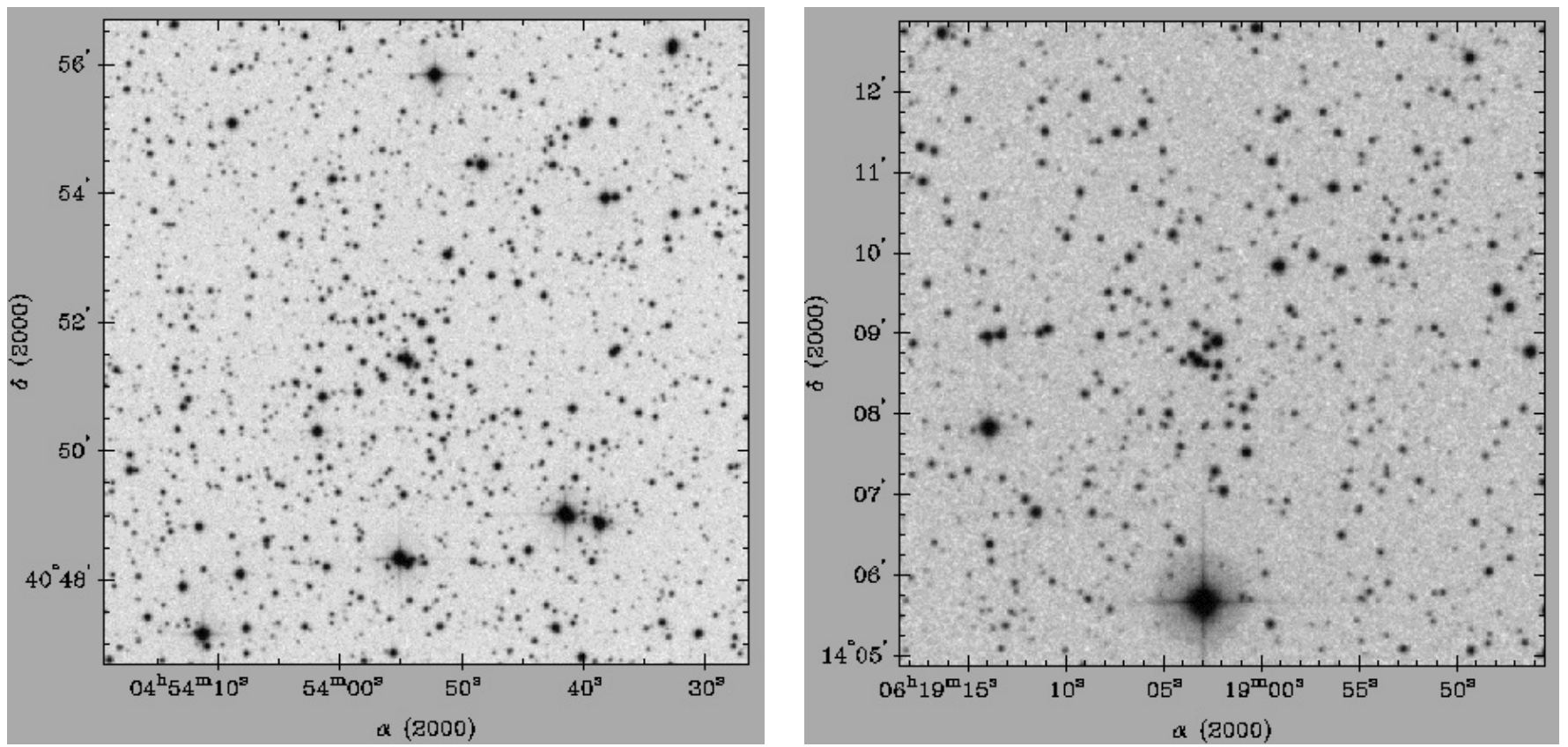

Fig. 1. Left panel: $10^{\prime} \times 10^{\prime}$ XDSS R image of FSR 735. Right panel: $10^{\prime} \times 10^{\prime}$ XDSS $R$ image of FSR 953. Images centred on the optimised coordinates.
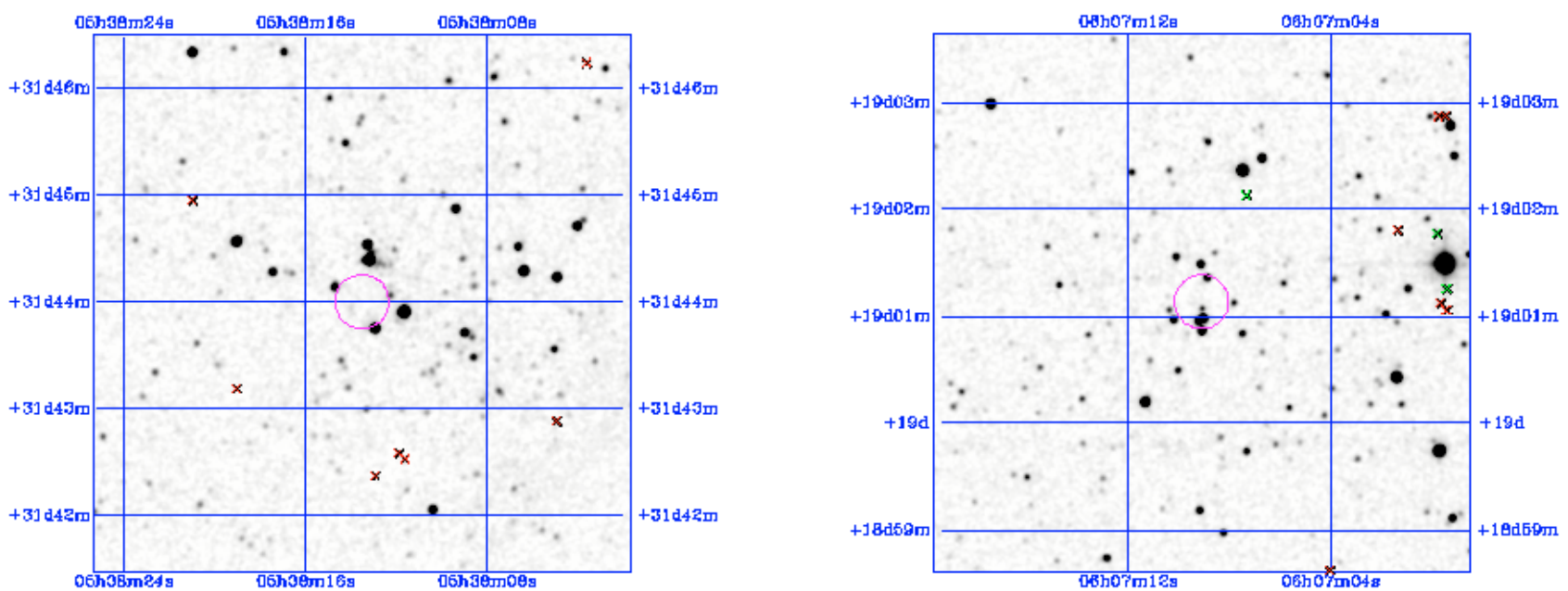

Fig. 2. Left panel: 2MASS $K$ image $5^{\prime} \times 5^{\prime}$ of FSR 812. Right panel: 2MASS $K$ image $5^{\prime} \times 5^{\prime}$ of FSR 904. Images centred on the optimised coordinates. The small circle indicates the cluster central region.

Table 2. Cross-identification of the open clusters.

\begin{tabular}{lrrrrr}
\hline \hline Desig\#1 & Desig\#2 & Desig\#3 & Desig\#4 & Ref. & Par. \\
$(1)$ & $(2)$ & $(3)$ & $(4)$ & $(5)$ & $(6)$ \\
\hline KKC1 & FSR 788 & Sh2-235 East1 & - & 2,4 & - \\
FSR 795 & Koposov 10 & - & - & 2,3 & 3 \\
Czernik 22 & Basel 4 & FSR 825 & OCI -455 & $1,2,3$ & - \\
FSR 828 & Koposov 43 & - & - & 2,3 & 3 \\
FSR 856 & Koposov 53 & - & - & 2,3 & 3 \\
Czernik 24 & FSR 881 & OCI -472 & - & $1,2,3$ & 3 \\
NGC 2234 & FSR 938 & - & - & 1,2 & - \\
\hline
\end{tabular}

Notes. Column (5) show references for cross-identification and Col. (6) references for parameters determination. The references are: 1 - Alter et al. (1970); 2 - Froebrich et al. (2007); 3 - Koposov et al. (2008); 4 - Kumar et al. (2006).
Some clusters are seen in visible bands (Fig. 1), while others are essentially infrared objects (Fig. 2).

\section{2MASS photometry}

$2 \mathrm{MASS}^{2}$ photometry (Skrutskie et al. 2006) in the $J, H$, and $K_{\mathrm{S}}$ bands was extracted in circular regions centred on the coordinates of the FSR objects using VizieR ${ }^{3}$. Wide extraction areas are essential for producing RDPs (Sect. 4) with a high contrast against the background and for a consistent field-star decontamination (Sect. 3.1). We started by assuming the FSR coordinates

2 The Two Micron All Sky Survey, available at www. ipac. caltech. edu/2mass/releases/allsky/

${ }^{3}$ http://vizier.u-strasbg.fr/viz-bin/VizieR? - source=II $/ 246$ 
Table 3. Field star decontamination statistics.

\begin{tabular}{|c|c|c|c|c|c|c|}
\hline Target & $\begin{array}{r}\left(R_{\max }\right) \\
\left({ }^{\prime}\right) \\
(2)\end{array}$ & $\begin{array}{r}N_{\mathrm{obs}} \\
\text { (stars) } \\
(3)\end{array}$ & $\begin{array}{r}N_{\mathrm{cl}} \\
\text { (stars) } \\
(4)\end{array}$ & $N_{1 \sigma}$ & $\begin{array}{r}\sigma_{\mathrm{FS}} \\
\text { (stars) } \\
(6)\end{array}$ & $F S_{\text {unif }}$ \\
\hline \multicolumn{7}{|c|}{ Confirmed OCs } \\
\hline $\mathrm{Cz} 22$ & 3 & $177 \pm 13$ & $93 \pm 3$ & 6.5 & 11.7 & 0.09 \\
\hline FSR 735 & 3 & $175 \pm 13$ & $84 \pm 3$ & 6.1 & 3.0 & 0.02 \\
\hline SR 80 & 4 & $150 \pm 12$ & $93 \pm 3$ & 7.1 & 4.8 & 0.04 \\
\hline FSR 812 & 3 & $92 \pm 10$ & $56 \pm 2$ & 5.7 & 2.2 & 0.03 \\
\hline FSR 826 & 5 & $423 \pm 21$ & $98 \pm 33$ & 4.8 & 8.7 & 0.02 \\
\hline FSR 8 & 4 & $359 \pm 19$ & $122 \pm 4$ & 6.3 & 10.5 & 0.04 \\
\hline & 5 & $196 \pm 14$ & $104 \pm 4$ & 7.1 & 6.5 & 0.05 \\
\hline FSR 9 & 3 & $99 \pm 10$ & $58 \pm 2$ & 5.8 & 3.8 & 0.07 \\
\hline & 5 & $387 \pm 20$ & $92 \pm 4$ & 3.9 & 24.4 & 0.07 \\
\hline & 3 & $141 \pm 12$ & $57 \pm 2$ & 4.3 & 6.8 & 0.06 \\
\hline NGC & 4 & $250 \pm 16$ & $84 \pm 4$ & 5.0 & 18.2 & 0.08 \\
\hline \multicolumn{7}{|c|}{ Uncertain cases } \\
\hline FSR 815 & 2 & $45 \pm 7$ & $32 \pm 1$ & 4.7 & 1.8 & 0.06 \\
\hline & 3 & $196 \pm 14$ & $62 \pm 4$ & 4.1 & 7.3 & 0.05 \\
\hline & 2 & $97 \pm 10$ & $42 \pm 2$ & 4.3 & 2.9 & 0.05 \\
\hline & 3 & $90 \pm 9$ & $44 \pm 2$ & 4.5 & 4.4 & 0.08 \\
\hline & 2 & $105 \pm 10$ & $50 \pm 2$ & 4.8 & 7.9 & 0.13 \\
\hline \multicolumn{7}{|c|}{ Possible field fluctuations } \\
\hline FSR 714 & 3 & $126 \pm 11$ & $34 \pm 3$ & 1.9 & 16.5 & 0.14 \\
\hline & 2 & $5 \pm 6$ & $24 \pm 1$ & 4.0 & 1.7 & 0.09 \\
\hline FSR 7 & 2 & $62 \pm 8$ & $22 \pm 1$ & 2.7 & 6.9 & 0.15 \\
\hline & 3 & $88 \pm 9$ & $37 \pm 2$ & 3.8 & 8.6 & 0.15 \\
\hline & 2 & $4 \pm 9$ & $33 \pm 1$ & 3.7 & 2.6 & 0.05 \\
\hline & 3 & $90 \pm 9$ & $37 \pm 3$ & 3.7 & 19.6 & 0.30 \\
\hline & 3 & $119 \pm 11$ & $35 \pm 2$ & 3.0 & 3.0 & 0.03 \\
\hline & 3 & $58 \pm 8$ & $27 \pm 1$ & 3.4 & 3.4 & 0.09 \\
\hline & 5 & $362 \pm 19$ & $81 \pm 4$ & 2.0 & 42.6 & 0.28 \\
\hline FS & 2 & $40 \pm 9$ & $29 \pm 1$ & 3.9 & 2.8 & 0.13 \\
\hline & 5 & $391 \pm 20$ & $81 \pm 5$ & 3.6 & 44.5 & 0.12 \\
\hline & 3 & \pm 14 & $53 \pm 2$ & 1.0 & 12.5 & 0.07 \\
\hline & 3 & $6 \pm 12$ & $54 \pm 3$ & 3.5 & 4.2 & 0.03 \\
\hline & 3 & 17 & $50 \pm 3$ & 3.6 & 9.1 & 0.07 \\
\hline $\mathrm{F}$ & 2 & $7 \pm 9$ & $35 \pm 2$ & 3.7 & 2.7 & 0.05 \\
\hline & 2 & $87 \pm 9$ & $36 \pm 2$ & 3.7 & 6.5 & 0.11 \\
\hline & 2 & $2 \pm 9$ & $38 \pm 2$ & 3.3 & 7.1 & 0.12 \\
\hline FS & 2 & $83 \pm 9$ & $36 \pm 2$ & 3.9 & 3.4 & 0.06 \\
\hline FSR 928 & 2 & $43 \pm 7$ & $10 \pm 1$ & 0.6 & 1.5 & 0.03 \\
\hline & 3 & $154 \pm 12$ & $50 \pm 3$ & 3.8 & 9.1 & 0.08 \\
\hline & 3 & $130 \pm 11$ & $35 \pm 3$ & 2.8 & 6.2 & 0.06 \\
\hline & 2 & $74 \pm 9$ & $29 \pm 2$ & 3.3 & 2.1 & 0.04 \\
\hline & 3 & $154 \pm 12$ & $46 \pm 3$ & 3.2 & 5.5 & 0.04 \\
\hline & 2 & $82 \pm 9$ & $36 \pm 2$ & 4.0 & 2.9 & 0.05 \\
\hline & 3 & $123 \pm 11$ & $18 \pm 2$ & 0.9 & 4.9 & 0.10 \\
\hline & 2 & $50 \pm 7$ & $28 \pm 1$ & 3.9 & 1.9 & 0.07 \\
\hline FSR 960 & 3 & $156 \pm 12$ & $52 \pm 3$ & 4.0 & 6.5 & 0.05 \\
\hline FSR 962 & 3 & $138 \pm 12$ & $30 \pm 3$ & 1.5 & 18.4 & 0.14 \\
\hline FSR 975 & 3 & $154 \pm 12$ & $46 \pm 3$ & 3.6 & 8.0 & 0.07 \\
\hline
\end{tabular}

Notes. The statistics of the full magnitude range covered by the respective CMD. CMDs extracted from $0 \lesssim R\left(^{\prime}\right) \lesssim R_{\max }$.

to centre the photometry extraction. Next, we computed the RDP (Sect. 4) to check cluster centring. In some cases the RDP built with the original FSR coordinates presented a dip at the centre. Then, new central coordinates are searched (after field-star decontamination - Sect. 3.1) to maximise the star-counts in the innermost RDP bin (e.g. Bonatto \& Bica 2009c).

\subsection{Field-star decontamination}

To uncover the intrinsic CMD morphology from the background stars, we applied the field-star decontamination procedure. This algorithm works on a statistical basis by measuring the relative number densities of probable cluster and field stars in cubic CMD cells that have axes along the $J,(J-H)$ and $\left(J-K_{\mathrm{s}}\right)$ magnitude and colours. These are the colours that provide the maximum distinction among CMD sequences for star clusters of different ages (e.g. Bonatto et al. 2004).

The algorithm (i) divides the full range of magnitude and colours of a given CMD into a 3D grid; (ii) computes the expected number-density of field stars in each cell based on the number of comparison field stars (within $1 \sigma$ Poisson fluctuation) with magnitude and colours compatible with those of the cell; and (iii) subtracts from each cell a number of stars that correspond to the number-density of field stars measured within the same cell in the comparison field. This method is sensitive to local variations in field star contamination with magnitude and colours. Cell dimensions are $\Delta J=1.0$, and $\Delta(J-H)=$ $\Delta\left(J-K_{\mathrm{s}}\right)=0.2$, which are adequate to allow sufficient starcount statistics in individual cells and preserve the morphology of the CMD evolutionary sequences. The dimensions of the colour/magnitude cells can be changed so that the total number of stars subtracted throughout the whole cluster area matches the expected one, within the $1 \sigma$ Poisson fluctuation. We provide here only a brief description of the decontamination procedure. For details see Bonatto \& Bica (2007a) and Bica et al. (2008a).

The decontamination algorithm provides the parameters $N_{\text {obs }}, N_{\text {cl }}, N_{1 \sigma}, \sigma_{\mathrm{FS}}$, and $F S_{\text {unif }}$ (Bica et al. 2008a), where $N_{\text {obs }}$ is the number of observed stars within the spatial region sampled in the CMD, $N_{\mathrm{cl}}$ represents the number of probable members after decontamination, and the parameter $N_{1 \sigma}$ gives a measure of the statistical significance of the decontaminated number of stars, and corresponds to the ratio of the number of stars in the decontaminated CMD with respect to the $1 \sigma$ Poisson fluctuation measured in the observed CMD. By definition, CMDs of overdensities must have $N_{1 \sigma}>1$. It is expected that CMDs of star clusters have integrated $N_{1 \sigma}$ that is significantly higher than 1 . The $N_{1 \sigma}$ values for the present sample are given in Col. 5 of Table 3, and $\sigma_{\mathrm{FS}}$ corresponds to the $1 \sigma$ Poisson fluctuation around the mean of the star counts measured in the 8 equal area sectors of the comparison field. Uniform comparison fields present low values of $\sigma_{\mathrm{FS}}$. Ideally, star clusters should have $N_{\mathrm{cl}}$ higher than $\sim 3 \sigma_{\mathrm{FS}}$. Finally, $F S_{\text {unif }}$ measures the star-count uniformity in the comparison field.

Since we usually work with comparison fields larger than the cluster extractions, the correction for the different spatial areas between field and cluster is expected to result in a fractional number of probable field stars $\left(n_{\mathrm{fs}}^{\text {cell }}\right)$ in some cells. Before the cell-by-cell subtraction, the fractional numbers are rounded off to the nearest integer, but limited to the number of observed stars in each cell $n_{\text {sub }}^{\text {cell }}=N I\left(n_{\mathrm{fs}}^{\text {cell }}\right) \leq n_{\text {obs }}^{\text {cell }}$, where NI represents rounding off to the nearest integer. The global effect is quantified by means of the difference between the expected number of field stars in each cell $\left(n_{\mathrm{fs}}^{\text {cell }}\right)$ and the actual number of subtracted stars $\left(n_{\text {sub }}^{\text {cell }}\right)$. Summed over all cells, this quantity provides an estimate of the total subtraction efficiency of the process,

$$
f_{\mathrm{sub}}=100 \times \sum_{\text {cell }} n_{\mathrm{sub}}^{\text {cell }} / \sum_{\text {cell }} n_{\mathrm{fs}}^{\text {cell }}(\%)
$$

Ideally, the best results would be obtained for an efficiency $f_{\text {sub }} \approx 100 \%$. In the present cases, the adopted grid settings produced subtraction efficiencies higher than 90\%. In Figs. 3-5 
Table 4. Derived fundamental parameters.

\begin{tabular}{|c|c|c|c|c|c|c|c|c|c|}
\hline Cluster & $\begin{array}{r}\alpha(2000) \\
(\mathrm{h} \mathrm{m} \mathrm{s})\end{array}$ & $\begin{array}{r}\delta(2000) \\
\left({ }^{\circ \prime \prime \prime}\right)\end{array}$ & $\begin{array}{r}E(B-V) \\
(\mathrm{mag})\end{array}$ & $\begin{array}{r}\text { Age } \\
(\mathrm{Myr})\end{array}$ & $\begin{array}{r}d_{\odot} \\
(\mathrm{kpc})\end{array}$ & $\begin{array}{r}R_{\mathrm{GC}} \\
(\mathrm{kpc})\end{array}$ & $\begin{array}{r}x_{\mathrm{GC}} \\
(\mathrm{kpc})\end{array}$ & $\begin{array}{r}y_{\mathrm{GC}} \\
(\mathrm{kpc})\end{array}$ & $\begin{array}{r}z_{\mathrm{GC}} \\
(\mathrm{kpc})\end{array}$ \\
\hline (1) & (2) & & (4) & (5) & (6) & (7) & $(8)$ & (9) & $(10)$ \\
\hline \multicolumn{10}{|c|}{ Confirmed OCs } \\
\hline $\mathrm{Cz} 22$ & 05:48:57 & $30: 10: 24$ & $0.64 \pm 0.03$ & $200 \pm 50$ & $2.6 \pm 0.1$ & $9.8 \pm 0.2$ & $-9.79 \pm 0.12$ & $+0.03 \pm 0.01$ & $-0.06 \pm 0.01$ \\
\hline FSR 735 & 04:53:52.9 & $40: 51: 42$ & $0.51 \pm 0.03$ & $500 \pm 100$ & $2.5 \pm 0.1$ & $9.6 \pm 0.1$ & $-9.56 \pm 0.10$ & $+0.67 \pm 0.03$ & $-0.08 \pm 0.01$ \\
\hline FSR 807 & $05: 36: 34.2$ & $31: 51: 20$ & $1.70 \pm 0.03$ & $5 \pm 3$ & $1.3 \pm 0.1$ & $8.5 \pm 0.1$ & $-8.5 \pm 0.06$ & $+0.08 \pm 0.01$ & $+0.00 \pm 0.01$ \\
\hline FSR 812 & $05: 38: 13.5$ & $31: 44: 00$ & $0.8 \pm 0.03$ & $10 \pm 5$ & $3.3 \pm 0.2$ & $10.5 \pm 0.2$ & $-11.3 \pm 0.20$ & $+0.19 \pm 0.01$ & $+0.01 \pm 0.01$ \\
\hline FSR 826 & $05: 42: 52.4$ & $28: 56: 29$ & $1.09 \pm 0.03$ & $10 \pm 5$ & $2.1 \pm 0.1$ & $9.3 \pm 0.1$ & $-9.27 \pm 0.01$ & $+0.01 \pm 0.01$ & $-0.02 \pm 0.01$ \\
\hline FSR 852 & $05: 53: 35$ & $25: 10: 52$ & $0.32 \pm 0.03$ & $1000 \pm 200$ & $2.2 \pm 0.1$ & $9.4 \pm 0.1$ & $-9.43 \pm 0.01$ & $-0.16 \pm 0.01$ & $-0.02 \pm 0.01$ \\
\hline FSR 904 & 06:07:09.1 & 19:01:08.2 & $0.64 \pm 0.03$ & $20 \pm 10$ & $2.2 \pm 0.1$ & $9.4 \pm 0.1$ & $-9.42 \pm 0.1$ & $-0.43 \pm 0.02$ & $-0.03 \pm 0.01$ \\
\hline FSR 941 & $06: 21: 47.3$ & $15: 44: 22.7$ & $0.80 \pm 0.03$ & $500 \pm 150$ & $5.8 \pm 0.3$ & $12.9 \pm 0.3$ & $-12.8 \pm 0.3$ & $-1.55 \pm 0.07$ & $+0.08 \pm 0.01$ \\
\hline FSR 953 & $\dagger: \dagger: \dagger$ & $\dagger: \dagger: \dagger$ & $0.48 \pm 0.03$ & $500 \pm 150$ & $2.6 \pm 0.2$ & $9.8 \pm 0.2$ & $-9.72 \pm 0.12$ & $-0.75 \pm 0.04$ & $-0.02 \pm 0.01$ \\
\hline FSR 955 & $06: 23: 56$ & $14: 30: 26$ & $0.51 \pm 0.03$ & $10 \pm 5$ & $3.7 \pm 0.2$ & $10.8 \pm 0.2$ & $-10.8 \pm 0.20$ & $-1.09 \pm 0.05$ & $+0.04 \pm 0.01$ \\
\hline NGC 2234 & $06: 29: 20$ & $16: 45: 27$ & $1.25 \pm 0.03$ & $50 \pm 20$ & $4.8 \pm 0.2$ & $11.9 \pm 0.2$ & $-11.88 \pm 0.2$ & $-1.29 \pm 0.06$ & $+0.24 \pm 0.01$ \\
\hline \multicolumn{10}{|c|}{ Uncertain cases } \\
\hline FSR 815 & $05: 37: 42.8$ & $31: 18: 19$ & & & & & & & \\
\hline FSR 883 & 06:04:26 & $21: 58: 54$ & & & & & & & \\
\hline FSR 902 & $06: 18: 20$ & $20: 30: 25$ & & & & & & & \\
\hline FSR 921 & $06: 05: 13.3$ & $16: 40: 54$ & & & & & & & \\
\hline FSR 951 & $\dagger: \dagger: \dagger$ & $\dagger: \dagger: \dagger$ & & & & & & & \\
\hline
\end{tabular}

Notes. Columns 2 and 3: Optimised central coordinates; $(\dagger)$ indicates same central coordinates as in Froebrich et al. (2007). Column 4: reddening in the cluster's central region. Column 5: age, from 2MASS photometry. Column 6: distance from the Sun. Column 7: $R_{\mathrm{GC}}$ calculated using $R_{\odot}=7.2 \mathrm{kpc}$ (Bica et al. 2006) as the distance of the Sun to the Galactic centre. Columns 8-10: Galactocentric components.

we show the spatial distribution of the stellar surface density for representative cases. This figure shows the surface density $(\sigma$, in units of stars $\operatorname{arcmin}^{-2}$ ) for a rectangular mesh with cells of dimensions $3^{\prime} \times 3^{\prime}$.

\subsection{Fundamental parameters}

The fundamental parameters are derived by means of the constraints provided by the field-decontaminated CMD morphology, especially the combined main sequence (MS) and pre-main sequence (PMS) star distribution, for young OCs (Fig. 7). The isochrones from the Padova group with solar metallicity (Girardi et al. 2002) computed with the 2MASS $J, H$, and $K_{\mathrm{s}}$ filters are used to represent the MS. Isochrones of Siess et al. (2000) are used to characterise the PMS sequences.

As summarised in Naylor \& Jeffries (2006), sophisticated approaches are available for analytical CMD fitting, especially the MS. However, given the poorly-populated MSs, the 2MASS photometric uncertainties for the fainter stars, the important population of PMS stars, and the differential reddening, we directly compared isochrones with the decontaminated CMD morphology. The fits are made by eye, taking the combined MS and PMS stellar distributions as constraints, and allowing for variations due to photometric uncertainties and differential reddening. Specifically, we start with the MS+PMS isochrone set for zero distance modulus and reddening, and next we apply magnitude and colour shifts until a satisfactory solution is reached. The young OCs of this sample present a significant fraction of stars redder than the youngest PMS isochrone. Most of this $\left(J-K_{\mathrm{S}}\right)$ excess towards the red is probably caused by differential reddening. The best fits are superimposed on decontaminated CMDs
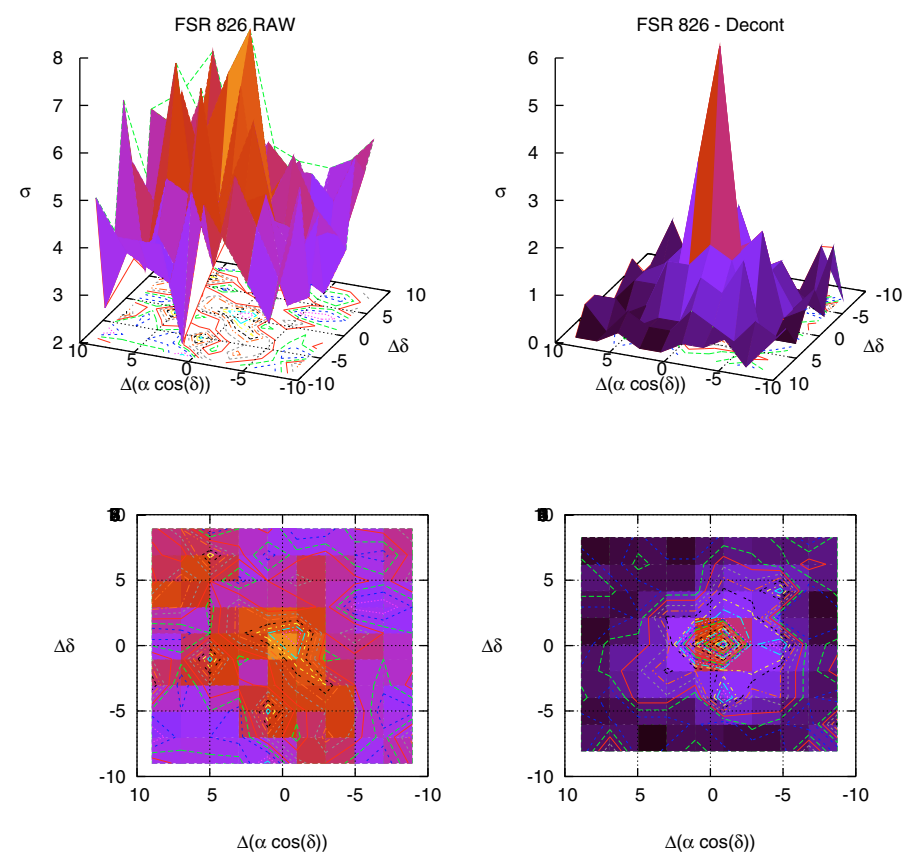

Fig. 3. Top panels: stellar surface-density $\sigma\left(\right.$ stars $\left.\operatorname{arcmin}^{-2}\right)$ of the confirmed OC FSR 826 computed for a mesh size of $3^{\prime} \times 3^{\prime}$, centred on the coordinates of this object (Tables 1 and 4). Bottom: the corresponding isopleth surfaces for observed (raw) photometry and decontaminated photometry.

(Figs. 6-8). The isochrone fit gives the observed distance modulus $(m-M)_{J}$ and reddening $E(J-H)$, which converts to $E(B-V)$ 

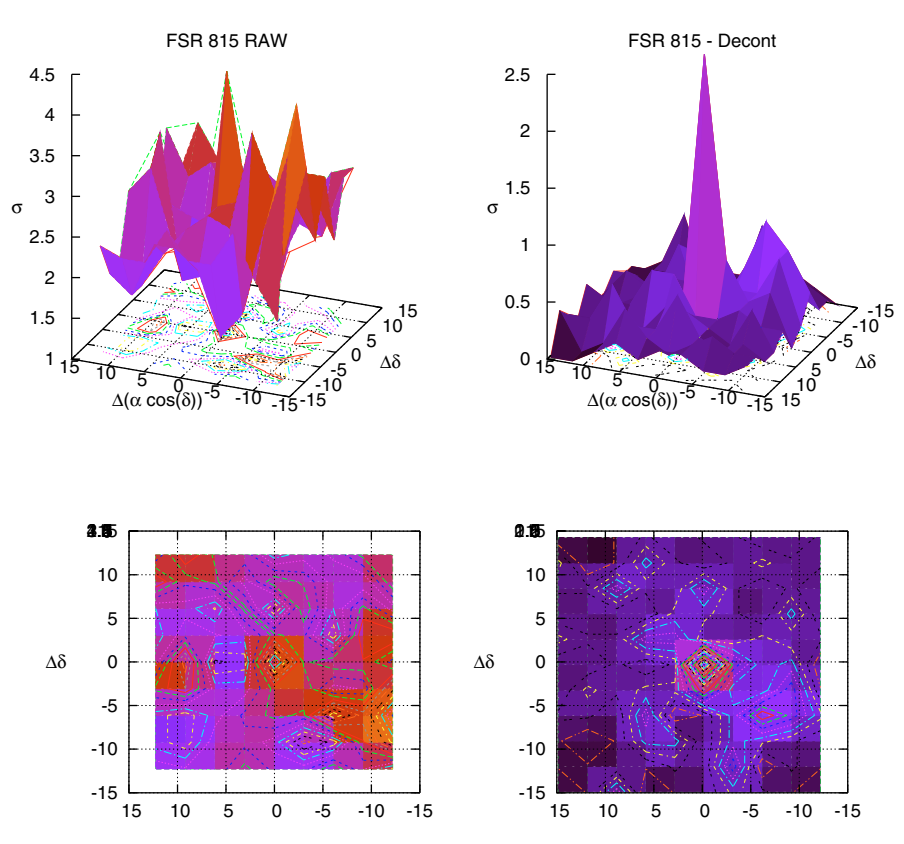

$\Delta(\alpha \cos (\delta))$

$\Delta(\alpha \cos (\delta))$

Fig. 4. Same as Fig. 3 for the uncertain case FSR 815.
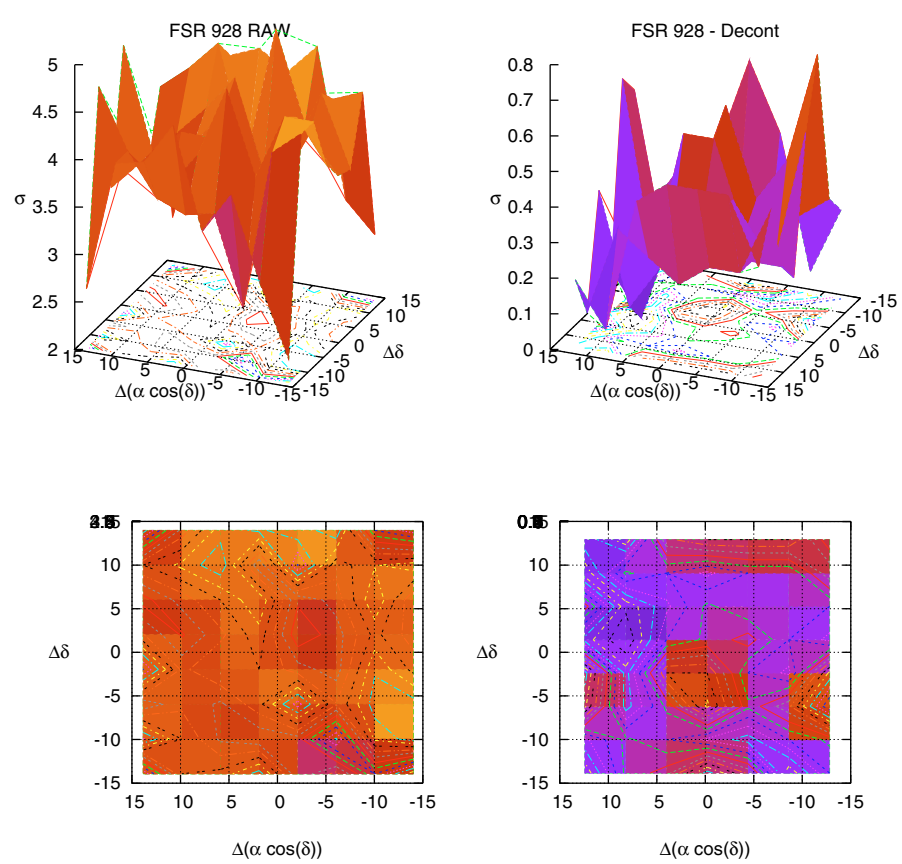

Fig. 5. Same as Fig. 3 for the possible field fluctuation case FSR 928.

and $A_{V}$ with the relations $A_{J} / A_{V}=0.276, A_{H} / A_{V}=0.176$, $A_{K_{\mathrm{s}}} / A_{V}=0.118, A_{J}=2.76 \times E(J-H)$, and $E(J-H)=$ $0.33 \times E(B-V)$ (Dutra et al. 2002), assuming a constant total-toselective absorption ratio $R_{V}=3.1$. We adopt the Sun's distance to the Galactic centre $R_{\odot}=7.2 \mathrm{kpc}$ (Bica et al. 2006) to compute the Galactocentric distance $\left(R_{\mathrm{GC}}\right)$. The resulting $E(B-V)$, age, $d_{\odot}$ and $R_{\mathrm{GC}}$ are given in Cols. 4 to 7 of Table 4 .

\subsection{Colour-colour diagrams}

Useful information on the nature of very young OCs can be obtained with colour-colour diagrams. Since our very young OCs

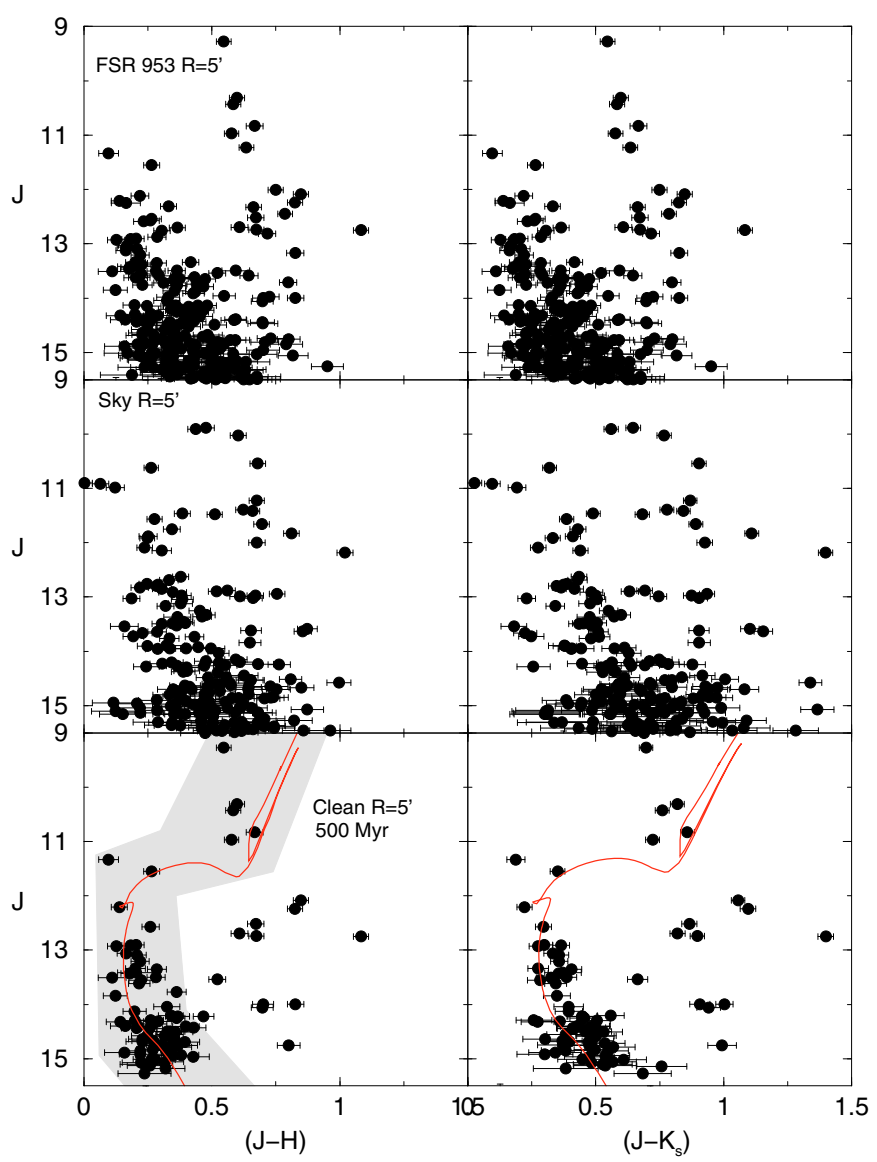

Fig. 6. 2MASS CMDs extracted from the $R=5^{\prime}$ region of FSR 953. Top panels: observed CMDs $J \times(J-H)$ (left) and $J \times\left(J-K_{\mathrm{s}}\right)($ right $)$. Middle panels: equal area comparison field. Bottom panels: field-star decontaminated CMDs fitted with the 500 Myr Padova isochrone (solid line) for FSR 953. The colour-magnitude filter used to isolate cluster MS/evolved stars is shown as a shaded region

include PMS stars, we show in Fig. 9 the decontaminated nearIR colour-colour diagram $\left(J-K_{\mathrm{s}}\right) \times\left(H-K_{\mathrm{s}}\right)$ of the member stars, together with tracks of Siess et al. (2000), set with the reddening values derived above, to characterise the age. If PMS stars are present in the cluster, it is expected that some stars present near-IR excess. As expected from the CMDs of very young candidates (Fig. 7), a significant number of the stars appear to be very reddened. Most stars have $\left(H-K_{\mathrm{s}}\right)$ colours close to the isochrone, within the uncertainties. Besides, most of the very red PMS stars are displaced parallel to the respective reddening vectors. However, few appear to present an abnormal excess in $\left(J-K_{\mathrm{S}}\right)$ and $\left(H-K_{\mathrm{s}}\right)$, especially FSR 807 , FSR 826 , and FSR 955, which may come from PMS stars still bearing circumstellar discs. The cluster can be reddened by foreground, circumcluster cloud, and/or dust around the PMS stars.

\section{Structural parameters}

Structural parameters are derived by means of the stellar radial density profile (RDP), which is the projected number of stars per area around the centre. RDPs are built with stars selected after applying the respective colour magnitude filter (CM filter) to the observed photometry. These tools isolate the most probable cluster sequences by excluding stars with discordant colours (e.g. Bonatto \& Bica 2007a, and references therein). However, residual field stars with colours similar to those of the cluster are 


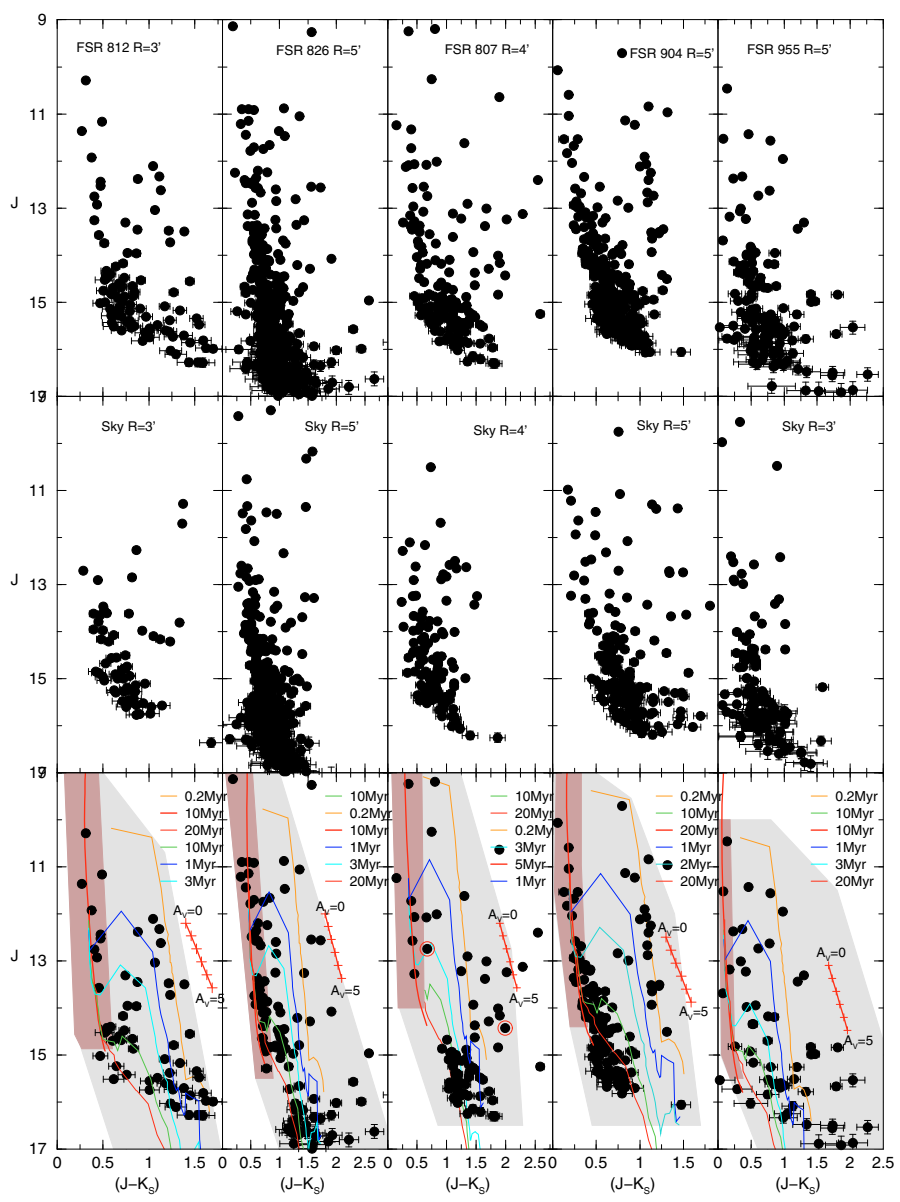

Fig. 7. 2MASS CMDs extracted from the central region of FSR 812, FSR 826, FSR 807, FSR904, and FSR 955. Top panels: observed CMDs $J \times\left(J-K_{\mathrm{s}}\right)$. Middle panels: equal area comparison field. Bottom panels: field-star decontaminated CMDs fitted with Padova isochrones (MS) stars and Siess PMS tracks. The colour-magnitude filters used to isolate cluster MS and PMS stars are shown as shaded regions. We also present the reddening vector for $A_{V}=0$ to $5 . \mathrm{H} \alpha$ emitters of FSR 807 are shown as circles around the stars.

expected to remain inside the $\mathrm{CM}$ filter region. They affect the intrinsic stellar RDP to a degree that depends on the relative densities of field and cluster stars. The contribution of these residual field stars to the RDPs is statistically quantified by means of a comparison field. In practical terms, the use of the CM filters in cluster sequences enhances the contrast of the RDP to the background. The CM filters are shown in Figs. 6-8 as the shaded region superimposed on the decontaminated CMDs.

To avoid oversampling near the centre and undersampling for large radii, the RDPs are built by counting stars in concentric rings of increasing width with distance to the centre. The number and width of rings are optimised so that the resulting RDPs have adequate spatial resolution with moderate $1 \sigma$ Poisson errors. The residual background level of each RDP corresponds to the average number of CM-filtered stars measured in the comparison field.

Usually, the RDPs of star clusters can be described by an analytical profile, like the empirical, single mass, modified isothermal spheres of King (1966) and Wilson (1975), and the power law with a core of Elson et al. (1987). These functions are characterised by different sets of parameters that are related to the cluster structure. For simplicity and with the RDP error bars (Fig. 11), we adopt the two-parameter function

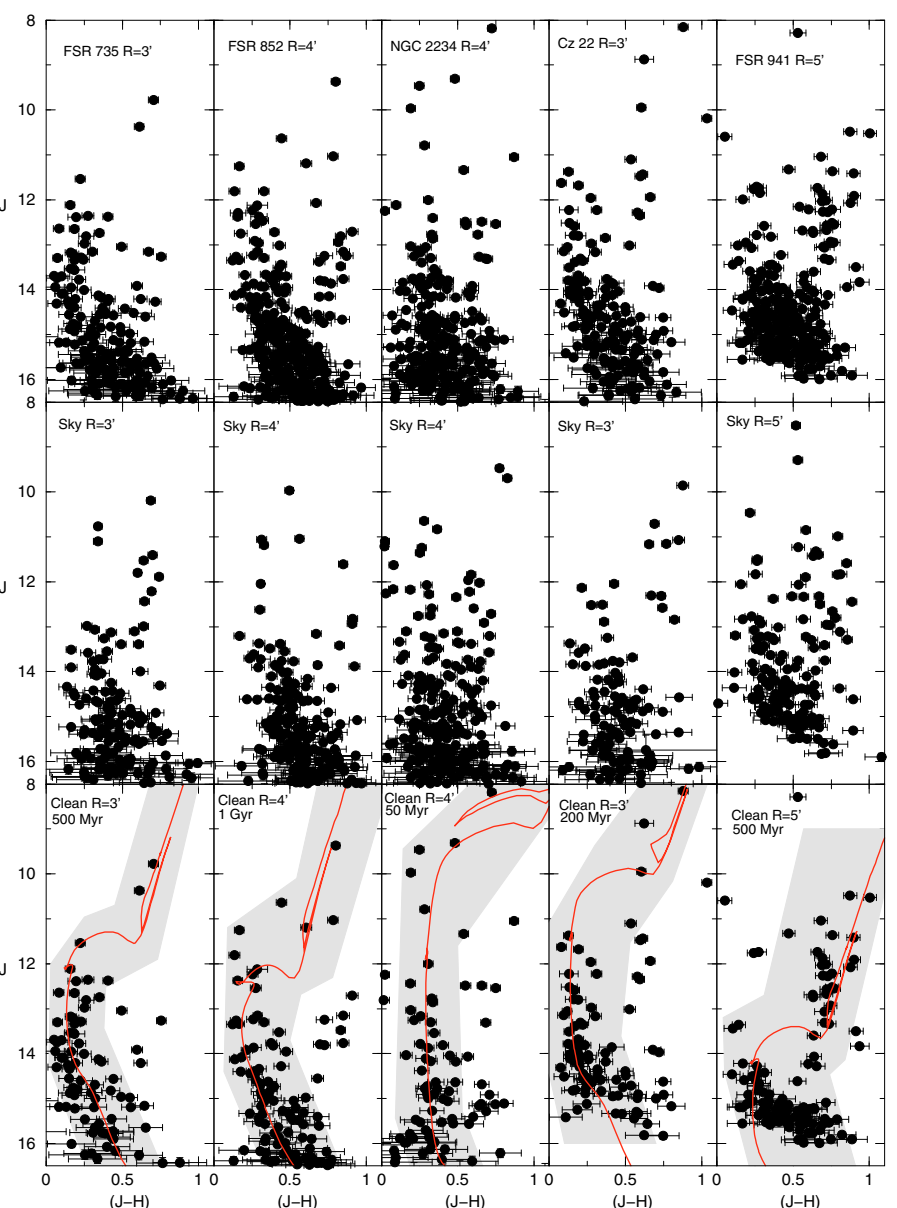

Fig. 8. 2MASS CMDs extracted from the central region of FSR 735, FSR 852, NGC 2234, Cz 22, and FSR 941. Top panels: observed CMDs $J \times(J-H)$. Middle panels: equal area comparison field. Bottom panels: field star decontaminated CMDs fitted Padova isochrone (solid line) for each OC. The colour-magnitude filter used to isolate cluster $\mathrm{MS} /$ evolved stars is shown as a shaded region.

$\sigma(R)=\sigma_{\mathrm{bg}}+\sigma_{0} /\left(1+\left(R / R_{\mathrm{c}}\right)^{2}\right)$, where $\sigma_{\mathrm{bg}}$ is the residual background density, $\sigma_{0}$ the central density of stars, and $R_{\text {core }}$ the core radius. Applied to star counts, this function is similar to that used by King (1962) to describe the surface brightness profiles in the central parts of globular clusters. We also estimate the cluster radius $\left(R_{\mathrm{RDP}}\right)$ by measuring the distance from the cluster centre where the RDP and residual background are statistically indistinguishable (e.g. Bonatto \& Bica 2007a). The $R_{\mathrm{RDP}}$ can be taken as an observational truncation radius, whose value depends both on the radial distribution of member stars and the field density.

\section{Results}

The overdensities are classified into three groups, according to the photometric and RDP analyses.

\subsection{Confirmed open clusters}

This group includes the objects with well-defined decontaminated CMD sequences (Figs. 6-8) with relatively high values of the parameter $N_{1 \sigma}$, as well as King-like RDPs (Fig. 11). For young OCs we also built colour-colour diagrams (Fig. 9). The astrophysical parameters could be measured for these objects (Tables 4 and 5). The previously unknown OCs are FSR 735, 


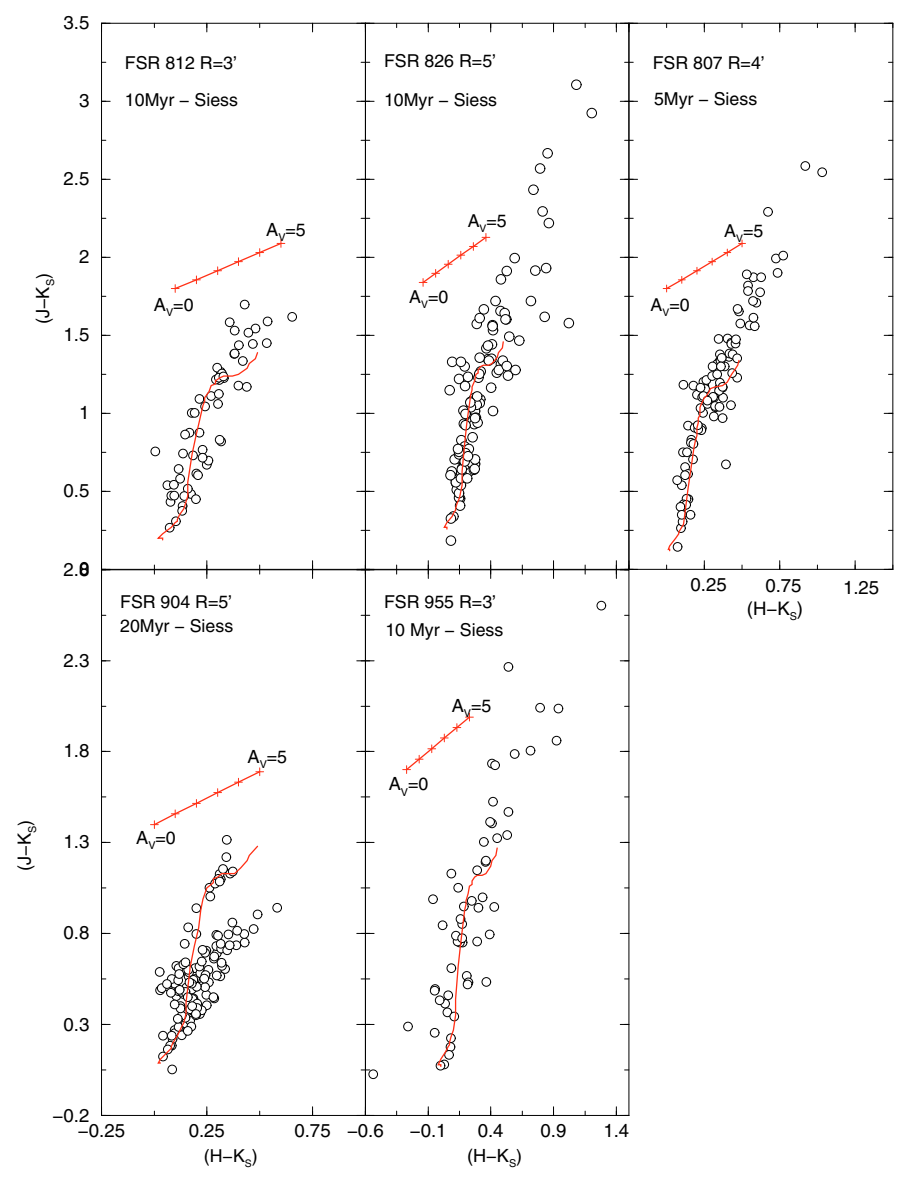

Fig. 9. Colour-colour diagrams with the decontaminated photometry of the young OCs in our sample, FSR 812, FSR 826, FSR 807, FSR 904, and FSR 955. Siess et al. (2000) isochrones and reddening vectors are used to characterise the PMS distribution.

FSR 807, FSR 812, FSR 826, FSR 852, FSR 904, FSR 941, FSR 953, and FSR 955. We also derived parameters for the previously catalogued $\mathrm{OCs} \mathrm{Cz} 22$ and NGC2234. KKC1 was confirmed as an OC, but the parameters of this object will be analysed in a forthcoming paper.

In Fig. 6 we present the $J \times(J-H)$ and $J \times\left(J-K_{\mathrm{s}}\right)$ CMDs extracted from a region $R=5^{\prime}$ centred on the optimised coordinates of the confirmed OC FSR 953 (top-panel). In the middle panels we show the comparison field corresponding to a ring with the same area as the central region. In the bottom panels we show the decontaminated CMDs with the 500 Myr Padova isochrones fitted. Figure 7 show $J \times\left(J-K_{\mathrm{s}}\right)$ CMDs for the confirmed young OCs FSR 812, FSR 826, FSR 807, FSR 904, and FSR 955. These objects present important populations of PMS stars and, therefore, we also use isochrones of Siess et al. (2000). To examine differential reddening, we include reddening vectors computed with the 2MASS ratios for visual absorptions in the range $A_{V}=0$ to 5 . We present in Fig. 8 the $J \times(J-H)$ CMDs for the remaining confirmed OCs FSR 735, FSR 852, NGC 2234, $\mathrm{Cz} 22$ and FSR 941. In Fig. 11, we present the RDPs of these objects, and in Table 5 we show the derived structural parameters. We show in Table 6 integrated colours and magnitudes for confirmed OCs.

Both FSR 904 and FSR 941 present a conspicuous excess over the King-like profile in the innermost RDP bin. This cusp has been detected in post-core collapse globular clusters (Trager et al. 1995) and some Gyr-old OCs, such as NGC 3960 (Bonatto

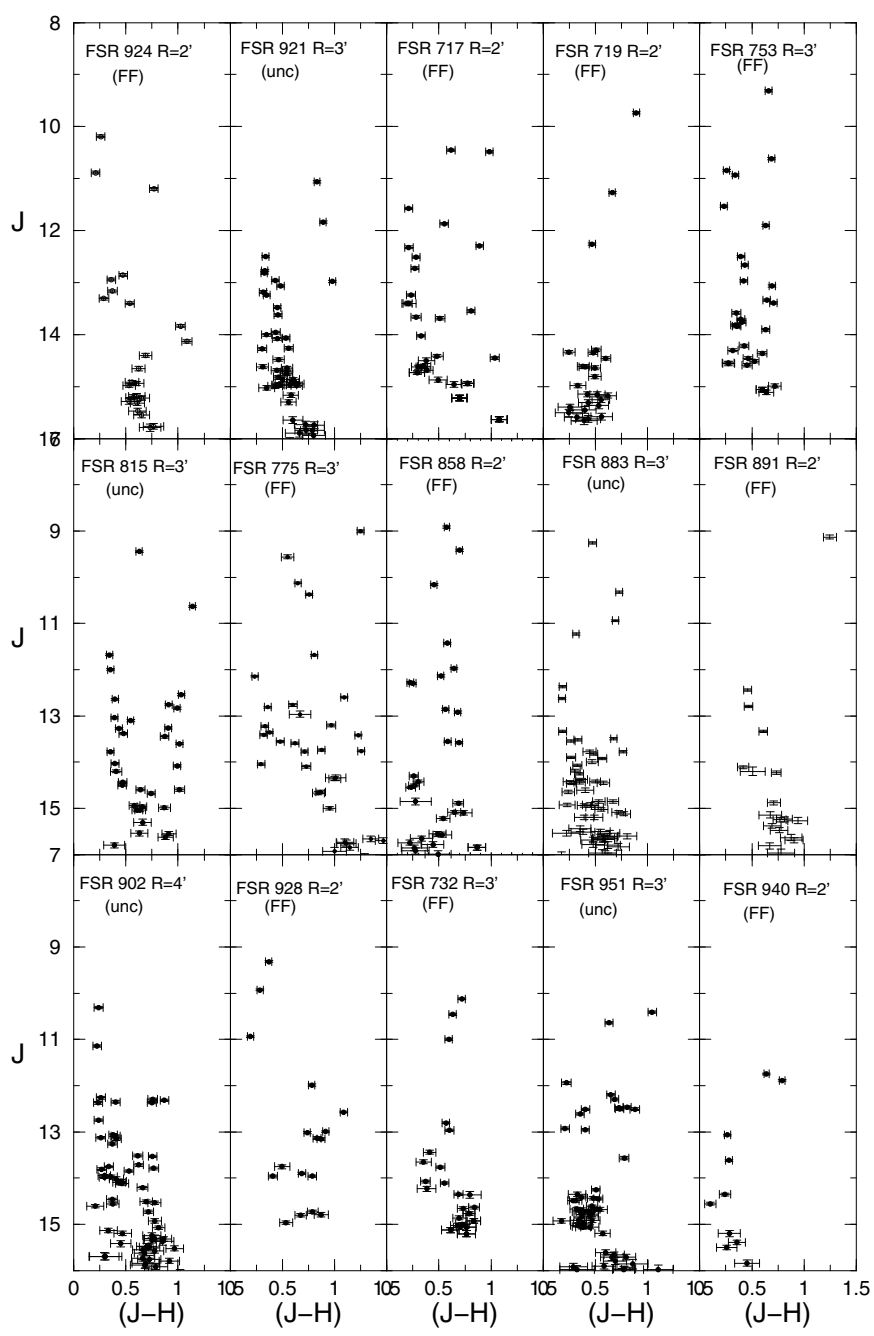

Fig. 10. Field-star decontaminated $J \times(J-H)$ CMDs of a representative sample of the uncertain cases (unc) and possible field fluctuations (FF).

\& Bica 2006) and LK 10 (Bonatto \& Bica 2009a). It has been attributed to advanced dynamical evolution. With $\sim 500 \mathrm{Myr}$ of age, FSR 941 is probably a core-collapsed OC. However, some very young OCs also present a cusp, probably as a consequence of molecular cloud fragmentation and/or star formation effects. In this context, we can mention NGC 2244 (Bonatto \& Bica 2009b), NGC 6823 (Bica et al. 2008b), Pismis 5 and NGC 1931 (Bonatto \& Bica 2009c), and FSR 198 (Camargo et al. 2009) as examples of young OCs with a central cusp. FSR 904 presents a similar effect.

Witham et al. (2008) presented the INT/WFC Photometric $\mathrm{H} \alpha$ Survey of the Northern Galactic Plane (IPHAS). This catalogue contains positions and photometry for 4853 sources with $\mathrm{H} \alpha$ excess (see also, Drew et al. 2005). The $\mathrm{H} \alpha$ emission is linked to several events in star clusters as stellar winds, T Tauri stars, Herbig-Haro objects, planetary nebulae and HII region associations. FSR 807 presents $2 \mathrm{H} \alpha$-excess sources within the cluster radius and 3 in the neighbourhood field $(J 053635.21+$ 31503.0 at $1.3^{\prime}$ of the cluster centre, $J 053631.98+314939.6$ at $1.7^{\prime}, J 053641.46+314627.8$ at $5.1^{\prime}, J 053619.82+314356.2$ at $8.0^{\prime}$, and $J 053619.58+314353.6$ at $\left.8.06^{\prime}\right)$ and FSR 812 presents 1 object $(J 053817.88+313934.8)$ at $4.5^{\prime}$ of the cluster centre. All objects are also present in the emission-line star catalogue of Kohoutek \& Wehmeyer (1999). We indicate the $2 \mathrm{H} \alpha$ emitters in FSR 807 CMD Fig. 7. 
Table 5. Structural parameters.

\begin{tabular}{|c|c|c|c|c|c|c|c|c|c|c|c|}
\hline uste & $\begin{array}{l}\left(1^{\prime}\right) \\
(\mathrm{pc})\end{array}$ & $\begin{array}{r}\sigma_{0 \mathrm{~K}} \\
\left(* \mathrm{pc}^{-2}\right)\end{array}$ & $\begin{array}{r}\sigma_{\mathrm{bg}} \\
\left(* \mathrm{pc}^{-2}\right)\end{array}$ & $\begin{array}{r}R_{\text {core }} \\
\text { (pc) }\end{array}$ & $\begin{array}{r}R_{\mathrm{RDP}} \\
(\mathrm{pc})\end{array}$ & $\begin{array}{r}\sigma_{0 \mathrm{~K}} \\
\left(*^{\prime-2}\right)\end{array}$ & $\begin{array}{r}\sigma_{\mathrm{bg}} \\
\left(*^{\prime-2}\right)\end{array}$ & $\begin{array}{r}R_{\text {core }} \\
\left(^{\prime}\right)\end{array}$ & $\begin{array}{r}R_{\mathrm{RDP}} \\
\left(^{\prime}\right)\end{array}$ & $\begin{array}{r}\Delta R \\
\left({ }^{\prime}\right)\end{array}$ & $\mathrm{CC}$ \\
\hline 1) & & (3) & (4) & (5) & (6) & (7) & (8) & (9) & (10) & (11) & (12) \\
\hline $\mathrm{Cz} 22$ & & $.8 \pm 4.7$ & $5 \pm 0.1$ & $53 \pm 0.07$ & $1 \pm 1.1$ & $1.42 \pm 2.6$ & $.93 \pm 0.04$ & $.72 \pm 0.1$ & $5.5 \pm 1.5$ & $10-20$ & 0.95 \\
\hline 51 & & 3.2 & .06 & & & & & & 1.0 & -30 & 0.95 \\
\hline & & 10.8 & \pm 0.3 & \pm 0.01 & & & .04 & & 0.5 & $20-40$ & 0.97 \\
\hline & & \pm 3.9 & \pm 0.1 & & & & & & 1.0 & $10-20$ & 0.91 \\
\hline & 59 & $8 \pm 8.6$ & 0.12 & & & & 0.04 & 0.1 & 1.5 & 40 & 0.96 \\
\hline & 54 & & & & & & & .27 & & & 0.90 \\
\hline & & & & & & & & & & & 0.95 \\
\hline & & & $.6 \pm 0.02$ & & & & & & 3.0 & $20-40$ & 0.80 \\
\hline & 0 & $75 \pm 5.7$ & $2.03 \pm 0.03$ & & & & & \pm 0.42 & $11.0 \pm 1.5$ & $20-30$ & 0.85 \\
\hline ESP 055 & 1.08 & $18.0 \pm 3.3$ & $3.6 \pm 0.05$ & $0.39 \pm 0.05$ & $3.2 \pm 1.1$ & $21.0 \pm 3.87$ & $4.28 \pm 0.06$ & $0.35 \pm 0.05$ & $3.0 \pm 1.0$ & $10-30$ & 0.97 \\
\hline NGC 2234 & 1.40 & $4.0 \pm 1.8$ & $0.9 \pm 0.01$ & $0.78 \pm 0.2$ & $5.6 \pm 1.4$ & $7.94 \pm 3.5$ & $1.73 \pm 0.03$ & $0.56 \pm 0.17$ & $4.0 \pm 1.0$ & $20-30$ & 0.91 \\
\hline
\end{tabular}

Notes. Column 2: arcmin to parsec scale. To minimise degrees of freedom in RDP fits with the King-like profile (see text), $\sigma_{\mathrm{bg}}$ was kept fixed (measured in the respective comparison fields) while $\sigma_{0}$ and $R_{\text {core }}$ were allowed to vary. Column 11: comparison field ring. Column 12: correlation coefficient.

Table 6. Integrated colours and magnitudes.

\begin{tabular}{|c|c|c|c|c|c|c|c|c|c|c|c|c|c|c|c|c|}
\hline \multirow{4}{*}{$\begin{array}{l}\text { Cluster } \\
\text { (1) }\end{array}$} & \multicolumn{6}{|c|}{ Magnitude } & \multirow{2}{*}{\multicolumn{2}{|c|}{$\frac{\text { Colour }}{\text { Reddening Corrected }}$}} & \multicolumn{8}{|c|}{ Half-light \& Starcount Radii } \\
\hline & \multicolumn{3}{|c|}{ Apparent } & \multicolumn{3}{|c|}{ Absolute } & & & \multicolumn{3}{|c|}{$R_{\mathrm{hl}}(\mathrm{pc})$} & \multicolumn{3}{|c|}{$R_{\mathrm{hl}}(')$} & \multirow{3}{*}{$\begin{array}{r}R_{\mathrm{sc}}(\mathrm{pc}) \\
(16)\end{array}$} & \multirow{3}{*}{$\begin{array}{r}R_{\mathrm{sc}}\left(^{\prime}\right) \\
\\
(17)\end{array}$} \\
\hline & $J$ & $H$ & $K_{\mathrm{s}}$ & $J$ & $H$ & $K_{\mathrm{s}}$ & $(J-H)$ & $\left(J-K_{\mathrm{s}}\right)$ & $J$ & $H$ & $K_{\mathrm{s}}$ & $J$ & $H$ & $K_{\mathrm{s}}$ & & \\
\hline & (2) & (3) & (4) & (5) & (6) & (7) & (8) & (9) & (10) & (11) & (12) & (13) & (14) & $(15)$ & & \\
\hline $\mathrm{Cz} 22$ & 7.5 & 6.9 & 6.7 & -5.1 & -5.5 & -5.6 & $46 \pm 0.29$ & $0.56 \pm 0.19$ & 0.8 & 0.8 & 0.8 & 1.1 & 1.1 & 1.0 & $1.6 \pm 0.2$ & $2.2 \pm 0.3$ \\
\hline FSR 735 & 8.0 & 7.4 & 7.3 & -5.3 & -5.4 & -5.3 & $17 \pm 0.03$ & $0.01 \pm 0.03$ & 2.4 & 2.4 & 2.4 & 3.4 & 3.4 & 3.4 & $1.1 \pm 0.2$ & $1.5 \pm$ \\
\hline FSR & 8.7 & 8.5 & 8.4 & -3.1 & -3.1 & -3.1 & $34 \pm 0.04$ & $0.50 \pm 0.03$ & 0.7 & 0.7 & 0.7 & 1.4 & 1.4 & 1.4 & $0.4 \pm 0.2$ & $0.8 \pm 0.5$ \\
\hline FSR 8 & 9.1 & 8.8 & 8.7 & -4.2 & -4.2 & -4.2 & $0.02 \pm 0.02$ & $0.00 \pm 0.02$ & 1.3 & 1.3 & 1.3 & 1.4 & 1.4 & 1.4 & $1.3 \pm 0.7$ & $1.4 \pm 0.7$ \\
\hline FSR & 9.5 & 9.0 & 8.8 & -3.0 & -3.1 & -3.2 & $5 \pm 0.02$ & $0.21 \pm 0.02$ & 1.6 & 1.6 & 1.6 & 2.7 & 2.7 & 2.7 & $=0.5$ & 2.4 \\
\hline & 8.1 & 7.6 & 7.4 & -3.9 & -4.3 & -4.5 & $43 \pm 0.04$ & $0.61 \pm 0.04$ & 5.0 & 5.0 & 5.0 & 7.8 & 7.8 & 7.8 & $2.8 \pm 0.8$ & $4.4 \pm$ \\
\hline & 7.8 & 7.7 & 7.4 & -4.5 & -4.4 & -4.6 & $0.01 \pm 0.02$ & $0.12 \pm 0.02$ & 2.4 & 2.4 & 2.4 & 3.7 & 3.7 & 3.7 & $5.6 \pm 0.6$ & $8.6 \pm 0.9$ \\
\hline & 10.5 & 10.2 & 10.0 & -4.0 & -4.1 & -4.2 & $0.05 \pm 0.13$ & $0.13 \pm 0.10$ & 1.3 & 1.2 & 1.2 & 0.8 & 0.7 & 0.7 & $9.7 \pm 0.8$ & $5.8 \pm 0.5$ \\
\hline FSR 953 & 8.6 & 8.2 & 8.1 & -3.9 & -4.1 & -4.1 & $0.35 \pm 0.03$ & $0.46 \pm 0.03$ & 2.3 & 2.3 & 2.3 & 3.0 & 3.0 & 3.0 & $4.0 \pm 1.3$ & $5.2 \pm 1.8$ \\
\hline FSR 955 & 12.0 & 11.8 & 10.8 & -1.3 & -1.3 & -2.2 & $0.03 \pm 0.24$ & $0.89 \pm 0.17$ & 0.8 & 0.8 & 1.4 & 0.8 & 0.7 & 1.3 & $0.7 \pm 0.4$ & $0.6 \pm 0.4$ \\
\hline NGC 2234 & 7.2 & 6.7 & 6.5 & -5.3 & -5.6 & -5.7 & $0.36 \pm 0.11$ & $0.44 \pm 0.28$ & 1.6 & 1.6 & 1.6 & 2.1 & 2.1 & 2.1 & $1.9 \pm 0.1$ & $2.5 \pm 0.1$ \\
\hline
\end{tabular}

Notes. Columns 2-4: apparent magnitude. Columns 5-7: absolute magnitude. Columns 8-9: reddening-corrected $(J-H)$ and $\left(J-K_{\mathrm{s}}\right)$ colours. Columns 10-15: half-light radii in pc and arcmin. Columns 16-17: star-count radii in pc and arcmin.

Table 7. Stellar mass estimate for clusters older than $50 \mathrm{Myr}$.

\begin{tabular}{|c|c|c|c|c|c|c|c|c|}
\hline \multirow[b]{2}{*}{ Cluster } & \multicolumn{6}{|c|}{ Observed in CMD } & \multicolumn{2}{|c|}{ Extrapolated } \\
\hline & $\begin{array}{c}\Delta m_{\mathrm{MS}} \\
\left(M_{\odot}\right) \\
(2)\end{array}$ & (3) & $\begin{array}{c}N_{\mathrm{MS}} \\
\text { (stars) } \\
(4)\end{array}$ & $\begin{array}{c}M_{\mathrm{MS}} \\
\left(M_{\odot}\right) \\
(5)\end{array}$ & $\begin{array}{c}N_{\text {evol }} \\
\text { (stars) } \\
(6)\end{array}$ & $\begin{array}{c}M_{\mathrm{evol}} \\
\left(M_{\odot}\right) \\
(7)\end{array}$ & $\begin{array}{c} \\
\text { (stars) } \\
(8)\end{array}$ & $\begin{array}{c}M \\
\left(M_{\odot}\right) \\
(9)\end{array}$ \\
\hline FSR 735 & $0.55-2.90$ & $1.21 \pm 0.11$ & $111 \pm 14$ & $164 \pm 20$ & $5 \pm 1$ & $15 \pm 3$ & $1200 \pm 889$ & $467 \pm 166$ \\
\hline FSR 852 & $0.65-2.10$ & $2.59 \pm 0.46$ & $132 \pm 19$ & $152 \pm 22$ & $4 \pm 6$ & $8 \pm 12$ & $2710 \pm 2190$ & $836 \pm 409$ \\
\hline FSR 953 & $0.85-2.50$ & $2.41 \pm 0.29$ & $108 \pm 14$ & $178 \pm 21$ & $8 \pm 3$ & $16 \pm 6$ & $5020 \pm 3850$ & $1670 \pm 719$ \\
\hline $\mathrm{Cz} 22$ & $1.30-3.90$ & $0.87 \pm 0.20$ & $60 \pm 10$ & $135 \pm 21$ & $5 \pm 3$ & $15 \pm 11$ & $1790 \pm 1290$ & $777 \pm 239$ \\
\hline FSR 941 & $1.70-2.90$ & $0.67 \pm 0.12$ & $147 \pm 13$ & $350 \pm 31$ & $40 \pm 15$ & $106 \pm 39$ & $13800 \pm 10200$ & $5480 \pm 1910$ \\
\hline NGC 2234 & $0.95-6.75$ & $0.97 \pm 0.10$ & $139 \pm 8$ & $431 \pm 28$ & $4 \pm 2$ & $32 \pm 18$ & $2640 \pm 1860$ & $1360 \pm 344$ \\
\hline
\end{tabular}

Notes. Column 2: MS mass range. Column 3: MS mass function slope $\chi$, derived from the fit of $\phi(m) \propto m^{-(1+\chi)}$. Columns 4-7: stellar content of the MS and evolved stars. Columns 8-9: stellar content extrapolated to $0.08 M_{\odot}$. 
Table 8. Stellar mass estimate for the clusters with PMS.

\begin{tabular}{|c|c|c|c|c|c|c|c|c|}
\hline \multirow[b]{2}{*}{ Cluster } & \multicolumn{4}{|c|}{ MS } & \multicolumn{2}{|c|}{ PMS } & \multicolumn{2}{|c|}{$\mathrm{MS}+\mathrm{PMS}$} \\
\hline & $\begin{array}{c}\Delta m_{\mathrm{MS}} \\
\left(M_{\odot}\right)\end{array}$ & $x$ & $\begin{array}{c}N \\
\text { (stars) }\end{array}$ & $\begin{array}{c}M \\
\left(M_{\odot}\right)\end{array}$ & $\begin{array}{c}N \\
\text { (stars) }\end{array}$ & $\begin{array}{c}M \\
\left(M_{\odot}\right)\end{array}$ & $\begin{array}{c}N \\
\text { (stars) }\end{array}$ & $\begin{array}{c}M \\
\left(M_{\odot}\right)\end{array}$ \\
\hline$(1)$ & (2) & (3) & (4) & $(5)$ & (6) & $(7)$ & (8) & (9) \\
\hline FSR 807 & $1.90-5.25$ & $-1.08 \pm 0.10$ & $8 \pm 3$ & $25 \pm 10$ & $21 \pm 6$ & $45 \pm 14$ & $29 \pm 9$ & $70 \pm 24$ \\
\hline FSR 812 & $6.75-13.0$ & $-0.34 \pm 0.15$ & $9 \pm 3$ & $83 \pm 30$ & $33 \pm 12$ & $135 \pm 35$ & $42 \pm 15$ & $218 \pm 65$ \\
\hline FSR 826 & $1.30-9.75$ & $1.07 \pm 0.12$ & $46 \pm 9$ & $178 \pm 31$ & $69 \pm 9$ & $172 \pm 43$ & $115 \pm 18$ & $350 \pm 74$ \\
\hline FSR 904 & $1.50-11.0$ & $0.26 \pm 0.16$ & $61 \pm 8$ & $188 \pm 42$ & $443 \pm 31$ & $706 \pm 61$ & $504 \pm 39$ & $894 \pm 103$ \\
\hline FSR 955 & $0.85-13.0$ & $0.22 \pm 0.09$ & $4 \pm 2$ & $20 \pm 7$ & $26 \pm 7$ & $109 \pm 35$ & $30 \pm 9$ & $129 \pm 42$ \\
\hline
\end{tabular}

Notes. Column 2: MS mass range. Column 3: MS mass function slope $\chi$, derived from the fit of $\phi(m) \propto m^{-(1+\chi)}$. Columns 4-7: stellar content of the MS and PMS stars. Columns 8-9: total (MS+PMS) stellar content.

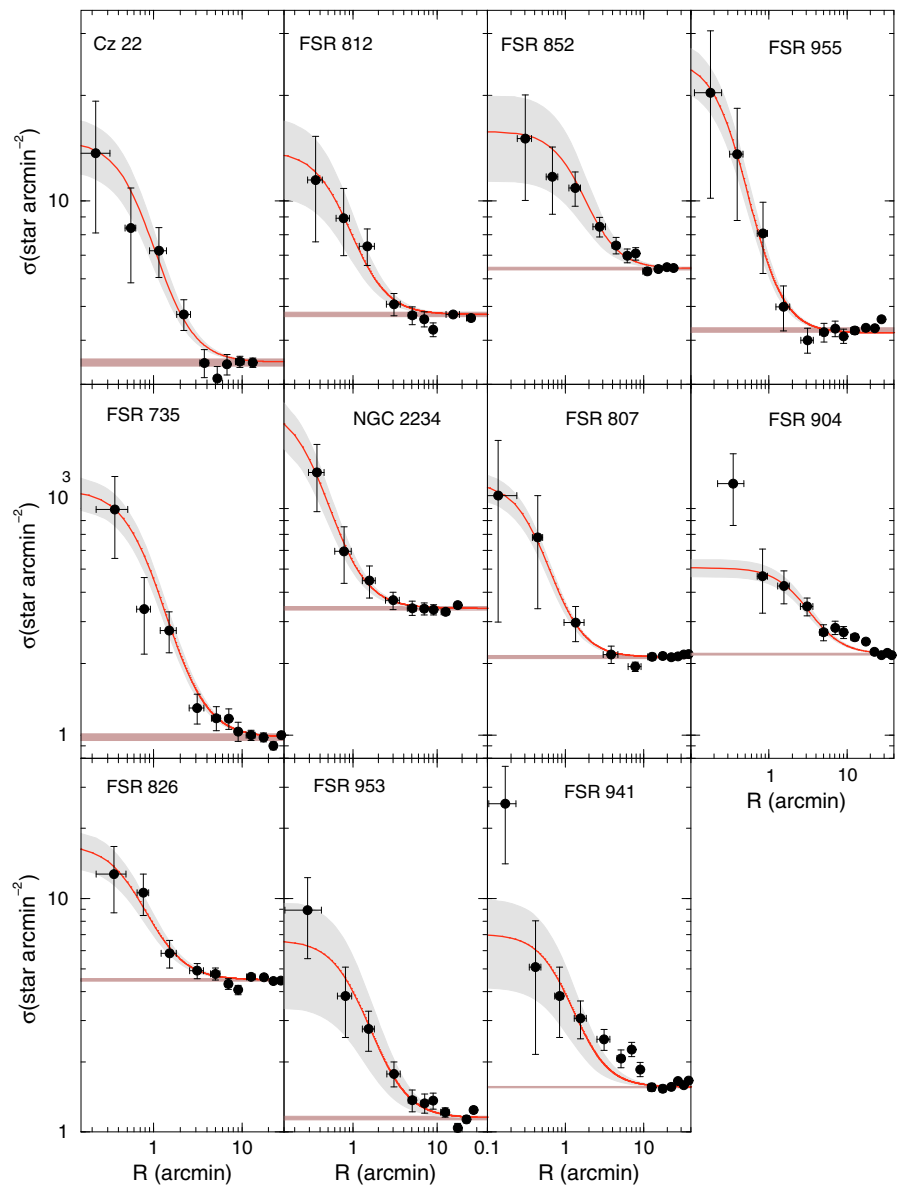

Fig. 11. Stellar RDPs (filled circles) built with colour-magnitude filtered photometry. Solid line: best-fit King profile. Horizontal shaded region: stellar background level measured in the comparison field. Grey regions: $1 \sigma$ King fit uncertainty.

\subsection{Uncertain cases}

The objects in this group have, in general, less defined decontaminated CMD sequences than those of the confirmed OCs, which is consistent with the lower level of the integrated $N_{1 \sigma}$ parameter. The irregular RDPs make difficult King's law fits. By "uncertain cluster", we mean those objects with a CMD that may suggest a cluster, but not the RDP (or the contrary). We suggest that deeper photometry, proper motions and other methods be employed to explore them in more detail. The uncertain cases

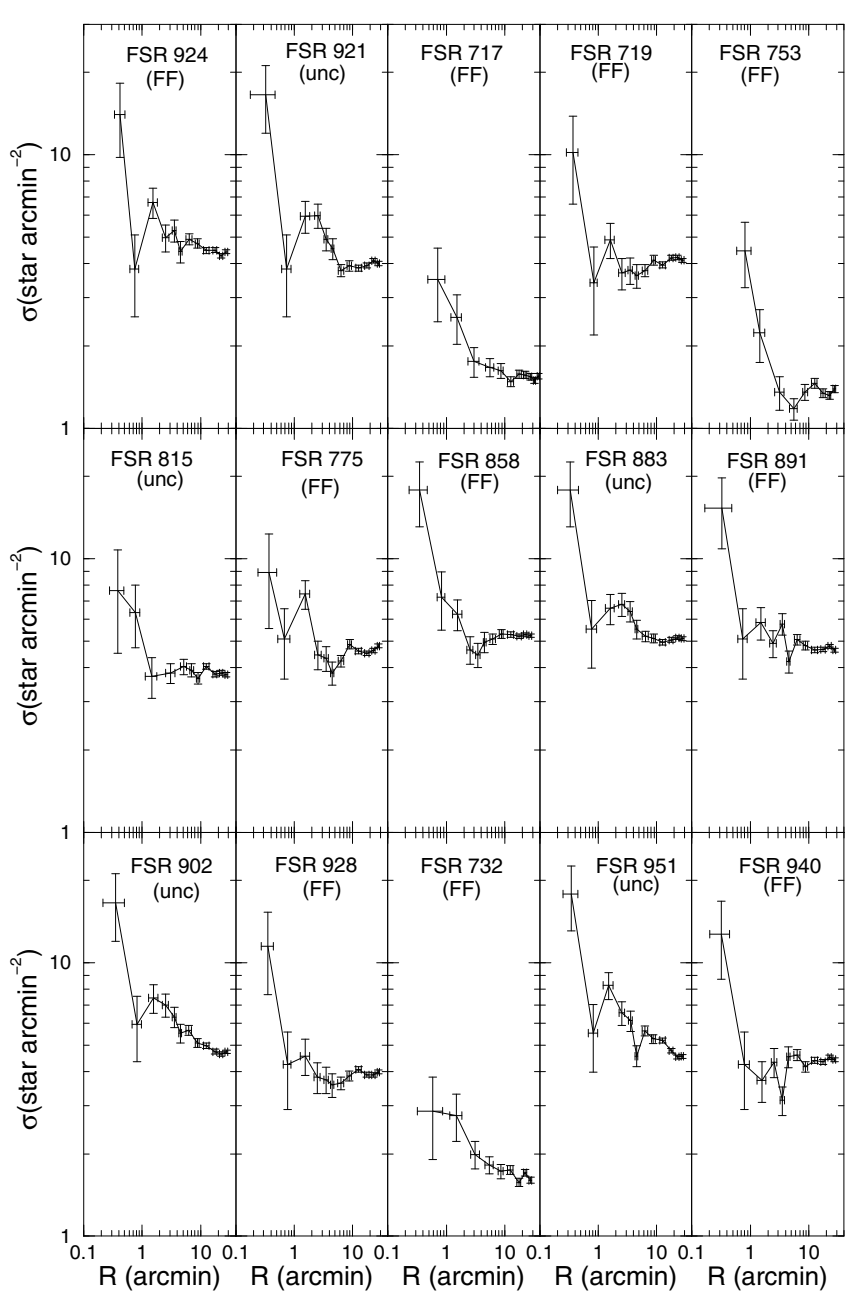

Fig. 12. RDPs of a representative sample of uncertain cases and probable field fluctuations.

are FSR 815, FSR 883, FSR 902, FSR 921, and FSR 951. In Fig. 10 we show the decontaminated $J \times(J-H)$ CMDs of a representative sample of the uncertain cases, and in Fig. 12 their RDPs.

\subsection{Possible field fluctuations}

Decontaminated CMDs of this group have $N_{1 \sigma}$-values significantly lower, and the RDPs much more irregular, than those of 
the other two groups (Sect. 3.1). In Fig. 10 we present the decontaminated $J \times(J-H)$ CMDs of a representative sample of the possible field fluctuations and Fig. 12 shows RDPs for some these overdensities.

\section{Mass estimate}

Given the somewhat limited 2MASS photometric depth and the relatively large distance (Table 5) of our confirmed OCs, the CMDs in Figs. 6-8 do not contain the whole mass range expected especially for OCs older than $\sim 50$ Myr. Thus, we estimate the stellar mass by means of the mass function (MF), built for the observed MS mass range according to Bica et al. (2006). The MS MF is then fitted with the function $\phi(m) \propto m^{-(1+\chi)}$. Details of this approach are given in Table 7 , where we also show the number and mass of the evolved stars. Clearly, we do not have access to the lower MS. Thus, assuming that the low-mass content is still present, we use Kroupa's (2001) $\mathrm{MF}^{4}$ to estimate the total stellar mass, down to the H-burning mass limit $\left(0.08 M_{\odot}\right)$. The results are given in the last two columns of Table 7. Interestingly, the extrapolation suggests that FSR 941 may be a relatively massive $\mathrm{OC}$.

For the young OCs we built the MS MF in a similar way to the old ones, and count the number of PMS stars (Table 8). Interestingly, the MF slopes are, in general, flatter than those of the older OCs (Table 7), which may reflect the longer timescale for the evolution towards the MS of the low-mass PMS stars. Given the differential reddening, it is not possible to attribute a precise mass value for each PMS star. Thus, we simply count the number of PMS stars and adopt an average mass value for the PMS stars to estimate $n_{\mathrm{PMS}}$ and $m_{\mathrm{PMS}}$. Assuming that the mass distribution of the PMS stars also follows Kroupa's (2001) MF, the average PMS mass - for masses within the range $0.08 \lesssim$ $m\left(M_{\odot}\right) \lesssim 7-$ is $<m_{\mathrm{PMS}}>\approx 0.6 M_{\odot}$. Thus, we simply multiply the number of PMS stars (Table 8) by this value to estimate the PMS mass. Finally, we add the latter value to the MS mass to obtain an estimate of the total stellar mass. Obviously, similarly to the MS stars, 2MASS cannot detect the very low mass PMS stars. Consequently, these values should be taken as lower limits.

\section{Discussion}

After cluster formation, structural parameters change with stellar and dynamical evolution. As a consequence of the rapid expulsion of primordial gas and the new lower gravitational potential, the cluster increases on all scales reaching for virialisation. Goodwin \& Bastian (2006) show that this early core radii expansion phase occurs within 10-30 Myr and can be explained as an effect of rapid residual gas expulsion. After gas expulsion (a few $10^{7} \mathrm{yr}$ ), when some energy equipartition is reached, the core radius of the OC survivors shrink, whereas the outer parts keep increasing in size. Mass loss due to stellar evolution also affects the structural, parameters but this effect is negligible because the most massive stars $\left(M_{\star}>30 M_{\odot}\right)$ hardly contribute to the mass of the cluster (Lamers \& Gieles 2006). In this context, Portegies Zwart et al. (1999) show that the maximum effect of stellar evolution on core radius expansion is about a factor two, but Mackey \& Gilmore (2008) show that this effect is more significant if the star cluster is initially mass segregated. They also show that significant core expansion due to stellar evolution occurs on timescales of $100 \mathrm{Myr}$.

\footnotetext{
${ }^{4} \chi=0.3 \pm 0.5$ for $0.08<m\left(M_{\odot}\right)<0.5, \chi=1.3 \pm 0.3$ for $0.5<m\left(M_{\odot}\right)<1.0$, and $\chi=1.3 \pm 0.7$ for $1.0<m\left(M_{\odot}\right)$.
}

As a consequence of large-scale mass segregation, massive stars tend to be more concentrated in the core of evolved clusters, while low-mass stars are transferred to the outer regions (Bonatto \& Bica 2005). As a consequence of mass segregation, $R_{\mathrm{RDP}}$ tends to increase, while $R_{\text {core }}$ decreases.

In Fig. 13 we compare the structural parameters derived for the present OCs with those measured in different environments. As a reference sample, we use $(i)$ some bright nearby OCs (Bonatto \& Bica 2005), including the two young OCs NGC 6611 (Bonatto et al. 2006a) and NGC 4755 (Bonatto et al. 2006b); (ii) OCs projected against the central parts of the Galaxy (Bonatto \& Bica 2007b); (iii) OCs projected close to the Galactic plane (Camargo et al. 2009); and (iv) the present sample.

In panel $(a)$ of Fig. 13, core and cluster radii of the OCs in sample $(i)$ are almost linearly related by $R_{\mathrm{RDP}}=(8.9 \pm 0.3) \times$ $R_{\text {core }}^{(1.0 \pm 0.1)}$, which suggests that both kinds of radii undergo a similar scaling, in the sense that, on average, larger clusters tend to have larger cores. However, $\frac{1}{3}$ of the OCs in sample (ii) do not follow that relation, which suggests that they are either intrinsically small or have been suffering important evaporation effects. The core and cluster radii in sample (iii) and the OCs of this work (iv) are consistent with the relation at the $1 \sigma$ level. A dependence of OC size on Galactocentric distance is shown in panel (b), as previously suggested by Lyngå (1982) and Tadross et al. (2002). The core and cluster radii of the OCs in this work (iv) are consistent with the one in samples (ii) and (iii). Most OCs of our sample are located in the inner disk and close to the spiral arms, so they are consistent with sample (iii) and with those located in crowded fields (ii). To explain the increase of Globular Cluster radii with Galactocentric distance, van den Bergh et al. (1991) suggest that part of the relation may be primordial, the higher molecular gas density in central Galactic regions may have created clusters with small radii. In addition, most clusters with small sizes are concentred near the Galactic plane, especially for $R_{\mathrm{GC}}<9.5 \mathrm{kpc}$ and $z_{\mathrm{GC}}<100 \mathrm{pc}$ (Tadross et al. 2002; Wielen 1971, 1975). FSR 807, which appears exceedingly small for its Galactocentric distance, possibly presents this primordial effect, with $z_{\mathrm{GC}}=0.0$ located close to Orion-Cygnus arm. In panels $(c)$ and $(d)$ we compare core and cluster radii with cluster age, respectively. This relationship is intimately related to cluster survival/dissociation rates. Both kinds of radii present a similar dependence on age, in which part of the clusters expand with time, while some seem to shrink. The bifurcation occurs at an age $\approx 1$ Gyr. Mackey \& Gilmore (2003) observed a similar effect for the core radii of LMC and SMC clusters and Mackey \& Gilmore (2008) argue that this radius-age correlation has a dynamical origin. They attribute the trend to slow contraction in $R_{\text {core }}$ to a dynamical relaxation and/or core collapse. The expansion was attributed to mass loss from rapid stellar evolution in a cluster that is mass-segregated or otherwise centrally concentrated and to heating due to a significant population of retained stellar mass black holes that are scattered into the cluster halo or ejected from the cluster (Mackey et al. 2007; Merritt et al. 2004). We also note that the radii of the clusters of our sample are related to the age similarly to the (leaky) ones of Pfalzner (2009) and optical clusters of Maciejewski \& Niedzielski (2007).

Most of the confirmed OCs of our sample are smaller than nearby OCs $(i)$ of similar age and Galactocentric distance (Fig. 13). We point out that our sample occurs close to the Galactic plane and/or close to spiral arms (except NGC 2234). Possibly, some of these OCs have interacted with GMCs. Molecular clouds more massive than $\sim 10^{6} M_{\odot}$ are found in the solar neighbourhood (Solomon et al. 1987). In Fig. 14 we show the relation between age and both $R_{\text {core }}$ and $\left|Z_{\mathrm{GC}}\right|$ for the 

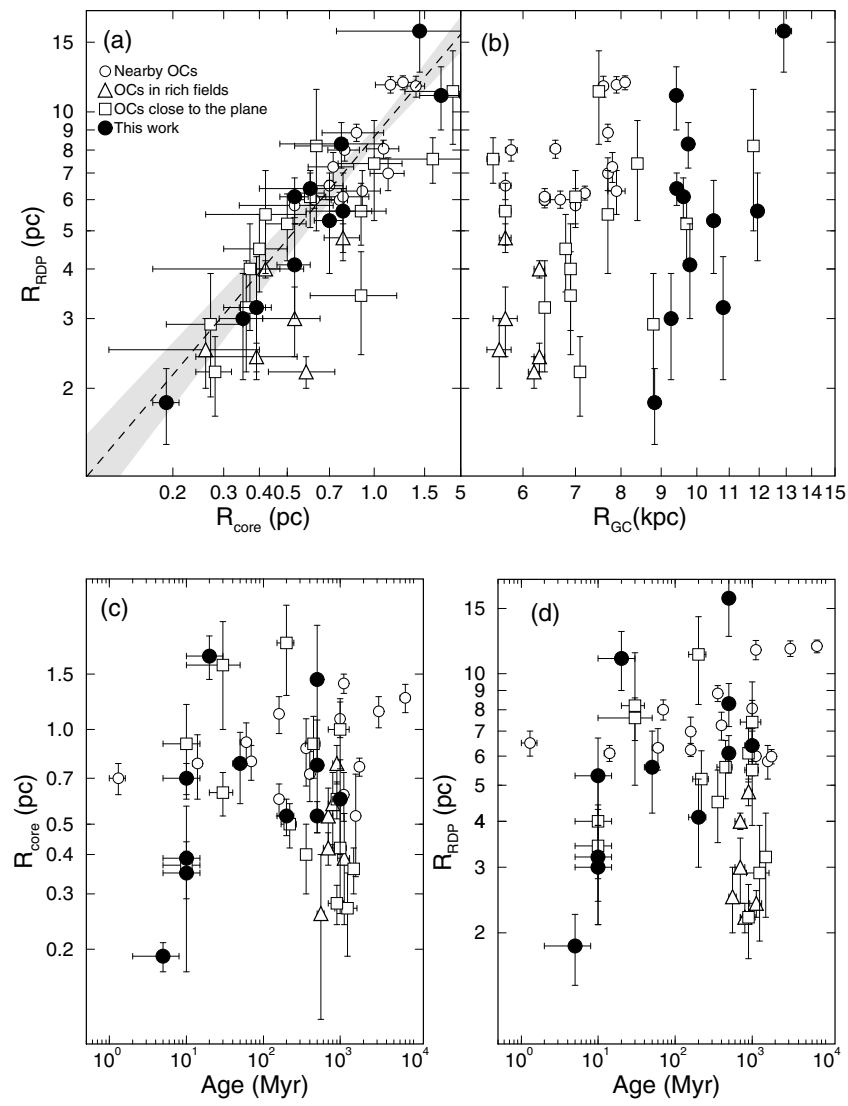

Fig. 13. Relations involving structural parameters of OCs. Empty circles: nearby OCs, including two young ones. Triangles: OCs projected on dense fields towards the Galactic central regions. Square: OCs close to the Galactic plane. Filled circles: the present work OCs.

young OCs of our sample. We fit an empirical curve $R_{\mathrm{c}}(\mathrm{pc})=$ $0.27 \times \ln ($ age $[\mathrm{Myr}])-0.25$ to young OCs close to the Galactic plane and/or close to spiral arms. Bastian et al. (2008) observed a similar relation for M 51 clusters and fitted the empirical curve $R_{\mathrm{c}}(\mathrm{pc})=0.6 \times \ln (\mathrm{age}[\mathrm{Myr}])-0.25$ that represents initially compact and mass-segregated star clusters $\left(R_{\text {core }} \approx 0.25 \mathrm{pc}\right)$. In this context, $N$-body simulations by Vesperini et al. (2009) show that in segregated clusters, early mass loss due to stellar evolution triggers a stronger expansion than for unsegregated clusters and that long-lived clusters initially with a high degree of mass segregation tend to have looser structure and reach core collapse later in their evolution than initially unsegregated clusters.

As discussed in Bonatto \& Bica (2009c), when the projected mass density of a star cluster follows a King-like profile (e.g. Bonatto \& Bica 2008a), the cluster mass $\left(M_{\text {clus }}\right)$ can be expressed as a function of the core radius and the central surface mass density $\left(\sigma_{\mathrm{M} 0}\right)$ according to $M_{\text {clus }} \approx 13.8 \sigma_{\mathrm{M} 0} R_{\mathrm{C}}^{2}$. Figure 14 (top-right panel) shows the distribution of our OCs in the plane core radius (Sect. 4) vs cluster mass (Sect. 6). Clearly, our OCs (together with the reference sample) distribute parallel to the above relation, since they are constrained within King-like distributions with $15 \lesssim \sigma_{\mathrm{M} 0}\left(M_{\odot} \mathrm{pc}^{-2}\right) \lesssim 600$. (These bounds take the uncertainties into account.) This suggests a correlation between cluster mass and core radius, somewhat consistent with the mass-radius relation suggested by Portegies Zwart et al. (2010) for massive clusters younger than $100 \mathrm{Myr}$.

Finally, with the cluster radius and mass estimates, we computed the cluster mass density $\rho\left(M_{\odot} \mathrm{pc}^{-3}\right)=\frac{3}{4 \pi} M_{\text {clus }} R_{\mathrm{RDP}}^{-3}$. We
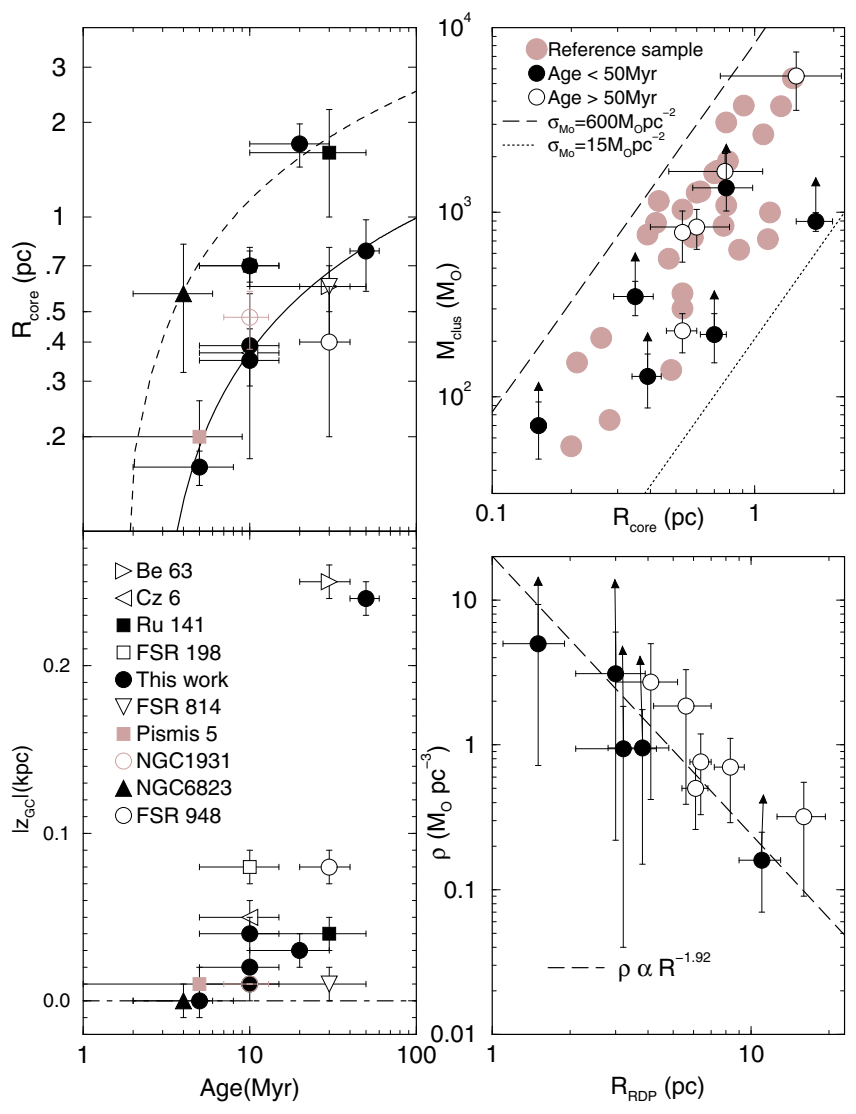

Fig. 14. Top-left panel: age and $R_{\text {core }}$ relations. The dashed line is the logarithmic fit of Bastian et al. $(2008)\left(R_{\text {core }}(\mathrm{pc})=0.6 \times \ln (\operatorname{age}[\mathrm{Myr}])-\right.$ $0.25)$ for M51 clusters. The solid line is our fit for Galactic clusters. Bottom-left panel: $\left|z_{\mathrm{GC}}\right|$ for clusters of the top panel. Top right: core radius and cluster mass follow the relation $M_{\text {clus }}=13.8 \sigma_{\mathrm{M} 0} R_{\mathrm{c}}^{2}$, with varying values of $\sigma_{\mathrm{M} 0}$; reference sample OCs are shown as grey circles. Filled circles show the OCs younger than 50 Myr, while the older ones are shown as open circles. Bottom right: cluster density vs. radius. Arrows indicate lower limits to the cluster mass and density.

show the results in the plane $R_{\mathrm{RDP}}$ vs. $\rho$ (Fig. 14, bottom-right panel). Despite the error bars, the density decreases with cluster radius as $\rho \propto R_{\mathrm{RDP}}^{-(1.92 \pm 0.36)}$, similarly to the sample of embedded clusters studied by Pfalzner (2009). That work, notes that the dependence on radius of the embedded clusters is significantly shallower than those observed in young clusters more massive than $10^{3} M_{\odot}, \rho \propto R^{-3}$ and $R^{-4}$. As a caveat, we note that the mass density of the OCs in Pfalzner (2009) is more than 10 times higher than those of our OCs.

Figure 15 shows the spatial distribution in the Galactic plane and spiral arms (Momany et al. 2006) of the present OCs, compared to that of the OCs in the WEBDA database. We considered two age ranges, $<1$ Gyr and $>1$ Gyr, and computed the projections on the Galactic plane of the Galactic coordinates $(\ell, b)$. Old OCs are mainly found outside the Solar circle, and the inner Galaxy contains few OCs so far detected. The interesting point is whether inner Galaxy clusters cannot be observed because of strong absorption and crowding, or have been systematically dissolved by the different tidal effects combined (Bonatto \& Bica 2007a, and references therein). In this context, the more OCs are identified (with their astrophysical parameters derived) in the central parts, the more constraints can be established to settle this issue. 


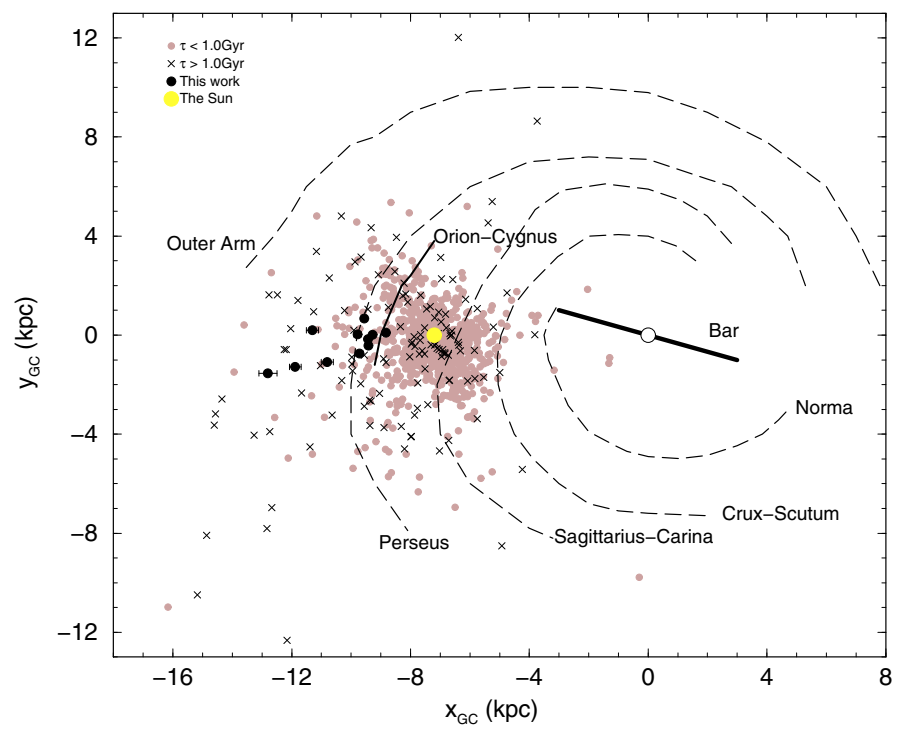

Fig. 15. Spatial distribution of the present star clusters (filled circles) compared to the WEBDA OCs with ages younger than 1 Gyr (brown circles) and older than $1 \mathrm{Gyr}$ (crosses). The schematic projection of the Galaxy is seen from the north pole, with $7.2 \mathrm{kpc}$ as the Sun's distance to the Galactic centre.

\section{Concluding remarks}

We investigate the nature of 50 overdensities projected nearly towards the anti-centre, in the sector $160^{\circ} \leq \ell \leq 200^{\circ}$, with $|b| \leq 20^{\circ}$ that were classified by Froebrich et al. (2007) as probable OCs and labelled with quality flags 2 and 3 . The candidates are analysed by means of 2MASS colour-magnitude diagrams, stellar radial density profiles, and colour-colour diagrams for young objects. Field-star decontamination is applied to uncover the cluster's intrinsic CMD morphology, and CM filters are used to disentangle probable cluster members.

Out of the 50 overdensities, $16(32 \%)$ are confirmed as OCs. Nine (18\%) are new OCs (FSR 735, FSR 807, FSR 812, FSR 826, FSR 852, FSR 904, FSR 941, FSR 953, and FSR 955) and we derived astrophysical parameters. They are OCs or embedded clusters with age in the range $5 \mathrm{Myr}$ to $1 \mathrm{Gyr}$, at distances from the Sun $1.28 \lesssim d_{\odot} \lesssim 5.78$ and Galactocentric distances $8.5 \lesssim R_{\mathrm{GC}} \lesssim 12.9$. Other 7 (14\%) overdensities are previously catalogued OCs or embedded clusters (KKC1, FSR 795, Cz 22, FSR 828, FSR 856, Czernik 24, and NGC 2234). We also derived parameters for $\mathrm{Cz} 22$ and NGC 2234. Five are classified as uncertain cases and require deeper photometry to establish their nature. The remaining FSR overdensities appear to be field fluctuations.

Most of the new OCs are located close to spiral arms and/or close to the Galactic plane and, probably because of this, the core radius appears to be smaller than the others at comparable Galactocentric distance and age. Also for this reason, most of them were undetected in the past.

Acknowledgements. We thank an anonymous referee for significant comments and suggestions. This publication makes use of data products from the Two Micron All Sky Survey, which is a joint project of the University of Massachusetts and the Infrared Processing and Analysis Centre/California Institute of Technology, funded by the National Aeronautics and Space Administration and the National Science Foundation. This research has made use of the WEBDA database, operated at the Institute for Astronomy of the University of Vienna, as well as Digitised Sky Survey images from the Space Telescope Science Institute obtained using the extraction tool from CADC (Canada). We acknowledge support from CNPq and Capes (Brazil).

\section{References}

Alter, G., Ruprecht, J., \& Vanysek, V. 1970, in Catalogue of star clusters and associations and Suppl., 2nd edn., Akad. Kiado, Budapest, ed. G. Alter, B. Balazs, \& J. Ruprecht

Bastian, N., Gieles, M., Goodwin, S. P., et al. 2008, MNRAS, 389, 223

Bergond, G., Leon, S., \& Guibert, J. 2001, A\&A, 377, 462

van den Bergh, S., Morbey, C., \& Pazder, J. 1991, ApJ, 375, 594

Bica, E., Bonatto, C., Barbuy, B., \& Ortolani, S. 2006, A\&A, 450, 105

Bica, E., Bonatto, C., \& Camargo, D. 2008a, MNRAS, 385, 349

Bica, E., Bonatto, C., \& Dutra, C. 2008b, A\&A, 489, 1129

Bonatto, C., \& Bica, E. 2005, A\&A, 437, 483

Bonatto, C., \& Bica, E. 2006, A\&A, 455, 931

Bonatto, C., \& Bica, E. 2007a, A\&A, 473, 445

Bonatto, C., \& Bica, E. 2007b, MNRAS, 377, 1301

Bonatto, C., \& Bica, E. 2008a, A\&A, 477, 829

Bonatto, C., \& Bica, E. 2008b, A\&A, 485, 81

Bonatto, C., \& Bica, E. 2009a, MNRAS, 392, 483

Bonatto, C., \& Bica, E. 2009b, MNRAS, 394, 2127

Bonatto, C., \& Bica, E. 2009c, MNRAS, 397, 1915

Bonatto, C., Bica, E., \& Girardi, L. 2004, A\&A, 415, 571

Bonatto, C., Santos, Jr., J. F. C., \& Bica, E. 2006a, A\&A, 445, 567

Bonatto, C., Bica, E., Ortolani, S., \& Barbuy, B. 2006b, A\&A, 453, 121

Camargo, D., Bonatto, C., \& Bica, E. 2009, A\&A, 508, 211

de Wit, W. J., Testi, L., Palla, F., \& Zinnecker, H. 2005, A\&A, 437, 243

Drew, J. E., Greimel, R., Irwin, M. J., et al. 2005, MNRAS, 362, 753

Dutra, C. M., Santiago, B. X., \& Bica, E. 2002, A\&A, 383, 219

Elson, R. A. W., Fall, S. M., \& Freeman, K. C. 1987, ApJ, 323, 54

Friel, E. D. 1995, ARA\&A, 33, 381

Froebrich, D., Scholz, A., \& Raftery, C. L. 2007, MNRAS, 374, 399

Gieles, M., Bastian, N., Lamers, H. J. G. L. M., \& Mout, J. N. 2005, A\&A, 441, 949

Gieles, M., Athanassoula, E., \& Portegies Zwart, S. F. 2007, MNRAS, 376, 809 Girardi, L., Bertelli, G., Bressan, A., et al. 2002, A\&A, 391, 195

Goodwin, S. P., \& Bastian, N. 2006, MNRAS, 373, 752

King I. 1962, AJ, 67, 471

King, I. 1966, AJ, 71, 64

Kohoutek, L., \& Wehmeyer, R. 1999, A\&AS, 134, 255

Kroupa, P. 2001, MNRAS, 322, 231

Kumar, M. S. N., Keto, E., \& Clerkin, E. 2006, A\&A, 449, 1033

Koposov, S. E., Glushkova, E. V., \& Zolotukhin, I. Yu. 2007, A\&A, 486, 771

Yadav, R. K. S., \& Sagar, R. 2004, MNRAS, 349, 1481

Lada, C. J., \& Lada, E. A. 2003, ARA\&A, 41, 57

Lada, E. A. 2007, Rev. Mex. Astron. Astrofis. Conf. Ser., 29, 43

Lamers, H. J. G. L. M., \& Gieles, M. 2006, A\&A, 455, 17

Lamers, H. J. G. L. M., Gieles, M., Bastian, N., et al. 2005, A\&A, 441, 117

Lyngå, G. 1982, A\&A, 109, 213

Maciejewski, G., \& Niedzielski, A. 2007, A\&A, 467, 1065

Mackey, A. D., \& Gilmore, G. F. 2003, MNRAS, 338, 120

Mackey, A. D., Wilkinson, M. I., Davies, M. B., \& Gilmore, G. F. 2007, MNRAS, 379, 40

Mackey, A. D., Gilmore, G. F., Davies, M. B., \& Gilmore, G. F. 2008, MNRAS, 386, 65

Merritt, D., Piatek, S., Portegies Zwart, S., \& Hemsendorf, M. 2004, ApJ, 608, 25

Momany, Y., Zaggia, S., Gilmore, G., et al. 2006, A\&A, 451, 515

Naylor, T., \& Jeffries, R. D. 2006, MNRAS, 373, 1251

Oort, J. H. 1957, in Stellar populations (Rome: Pontifical Academy of Science) discussion in session on star clusters

Pavani, D. N., \& Bica, E. 2007, MNRAS, 468, 139

Pfalzner, S. 2009, A\&A, 498, 37

Portegies Zwart, S. F., Makino, J., McMillan, S. L. W., \& Hut, P. 1999 A\&A, 348, 117

Portegies Zwart, S. F., McMillan, S., \& Gieles, M. 2010, ARA\&A,

[arXiv: 1002.1961]

Schilbach, E., Kharchenko, N. V., Pisnukov, A. E., Röser, S., \& Scholz, R.-D. 2006, A\&A, 456, 523

Siess, L., Dufour, E., \& Forestini, M. 2000, A\&A, 353, 593

Skrutskie, M. F., Cutri, R. M., Stiening, R., et al. 2006, AJ, 131, 1163

Solomon, P. M., Rivolo, A. R., Barrett, J., \& Yahil, A. 1987, ApJ, 319, 730

Trager, S. C., King, I. R., \& Djorgovski, S. 1995, AJ, 109, 218

Tadross, A. L., Werner, P., Osman, A., \& Marie, M. 2002, NewAst, 7, 553

Tutukov, A. V. 1978, A\&A, 70, 57

Vesperini, E., McMillan, S. L. W., \& Portegies Zwart, S. 2009 ApJ, 698, 615

Wielen, R. 1971, A\&A, 13, 309

Wielen, R. 1975, ed. A. Hayli (Dordrecht: Reidel), IAU symp., 69, 119

Wilson, C. P. 1975, AJ, 80, 175

Witham, A. R., Knigge, C., Drew, J. E., et al. 2008, MNRAS, 384, 1277 